IFT-P.016/2008

\title{
Yang-Mills Chern-Simons Corrections From The Pure Spinor Superstring
}

\author{
Oscar A. Bedoya国 \\ Instituto de Física Teórica, State University of São Paulo \\ Rua Pamplona 145, 01405-900, São Paulo, SP, Brasil.
}

\begin{abstract}
Nilpotency of the pure spinor BRST operator in a curved background implies superspace equations of motion for the background. By computing one-loop corrections to nilpotency for the heterotic sigma model, the Yang-Mills Chern-Simons corrections to the background are derived.
\end{abstract}

July 2008

1 e-mail: abedoya@ift.unesp.br

2 New address since August, 2008: Instituto de Física, Universidade de São Paulo, 05315-970, São Paulo, SP, Brasil. e-mail:abedoya@fma.if.usp.br 


\section{Introduction}

It is a well known fact that in order to couple the Type I or Heterotic superstrings to a generic background, the gauge groups must be $S O(32)$ or $E_{8} \times E_{8}$ in order to have a theory free of gauge and Lorentz anomalies. This condition is supplemented with an $\alpha^{\prime}$ correction to the 3 -superform $H$, defined as the exterior derivative of the Kalb-Ramond 2-superform B. The mechanism described is known as the Green-Schwarz mechanism [1] and the form of the corrections are of Yang-Mills and Lorentz Chern-Simons type, which is related to the form of the counter-terms that cancel the anomalies. It is worth to note that this mechanism for the cancellation of anomalies was discovered using the low energy limit of superstrings. However, Hull and Witten [2] noted the necessity of the Chern-Simons modifications in order to cancel the sigma model for the Heterotic superstring.

To describe superstrings in a generic background, one has at disposal the RamondNeveu-Schwarz (RNS) formalism and the Green-Schwarz (GS) formalism. However in the first, whose sigma model was the one used in [2], it is difficult to incorporate space-time fermions, so some elements are lacking; while in the second one can only quantize in the light-cone gauge, loosing the manifest symmetries. Nevertheless, there is one more description known as the Pure Spinor (PS) formalism [3], in which a superstring can be described in a generic background [4 and does not suffer of those difficulties. The quantization of the superstring in the PS formalism is performed through a BRST charge $Q_{B R S T}$, which is nilpotent because of the pure spinor condition, to be defined later on. As shown in [4], the classical BRST invariance impose some constraints on the background fields, in particular on the components of $H$; putting them on-shell. Before pure spinors were used to describe superstrings, integrability along pure spinor lines allowed to find the super Yang-Mills and supergravity equations of motion in ten dimensions [5]. Because of its nature, the pure spinor sigma model is a proper description for performing perturbative computations. Using this description it has been possible to compute the beta functions for the Heterotic [6] and Type II Superstring [7], showing that the classical BRST invariance implies in the conformal invariance 3 .

Because in the PS formalism one can quantize in a Super-Poincare invariant manner, one could attempt to compute $\alpha^{\prime}$ corrections to the constrains in the background fields mentioned in the last paragraph. In particular, one can look for Chern-Simons type corrections to the 3 -superform $H$ as mentioned in the first paragraph. This paper is concentrated

3 For further studies of the pure spinor formalism in a curved background see [8] 
in the Yang-Mills Chern-Simons correction to $H$, which was also shown in [9] and [10] to imply the correct coupling of $\mathcal{N}=1$ supergravity to $\mathcal{N}=1$ super Yang-Mills. Specifically, it will be computed corrections to the classical constraints on $H$ by checking the nilpotency of the BRST charge at one-loop level. It will be shown that it is a key aspect to add local counter-terms in the action to preserve the BRST invariance at the quantum level. Those counter-terms amounts to redefinitions of the space-time metric and the spin connection. The redefinition of the space-time metric was noted by Sen [11]. Furthermore Hull and Townsend [12] showed that they were necessary to preserve the world sheet supersymmetry in the heterotic string. Since the supervielbein $E_{M}{ }^{\alpha}(Z)$ appears as one of the superfields in the pure spinor sigma model, redefinitions of this superfield are in accordance with redefinition of the space-time metric, and as will be shown, they are important to check the BRST invariance at one-loop.

The structure of this paper is as follows. In section 2 a brief introduction to the PS formalism is given. In section 3 the results of [4] and [13] concerning the nilpotency of $Q_{B R S T}$ and holomorphicity of the BRST current at the lowest order in $\alpha^{\prime}$ are reobtained, by performing a tree-level computation. In section 4 it is performed a one-loop computation to find the Yang-Mills Chern-Simons correction to the 3 -superform $H$, explaining the computations in a detailed way, as well as the counter-terms introduced. In section 5 the work is concluded. In the appendix are included the results of the background field expansion used in the computation.

\section{Review of the Pure Spinor Formalism}

The action for the heterotic superstring in the pure spinor formalism [3] is given by

$$
S=\frac{1}{2 \pi \alpha^{\prime}} \int d^{2} z\left(\frac{1}{2} \partial X^{m} \bar{\partial} X_{m}+p_{\alpha} \bar{\partial} \theta^{\alpha}+\bar{b} \partial \bar{c}\right)+S_{\lambda}+S_{\bar{J}}
$$

where the worldsheet variables $\left(X^{m}, \theta^{\alpha}, p_{\alpha}\right)$, with $m=0 \ldots 9, \alpha=1 \ldots 16$, describe the $N=1 D=10$ superspace. $p_{\alpha}$ is the conjugate momentum to $\theta^{\alpha}$. This formalism takes its name from the bosonic spinor $\lambda^{\alpha}$, which is constrained to satisfy the pure spinor condition $\lambda^{\alpha}\left(\gamma^{m}\right)_{\alpha \beta} \lambda^{\beta}=0$, where $\gamma^{m}$ are $16 \times 16$ symmetric ten-dimensional gamma matrices. The pure spinor part of the action, denoted by $S_{\lambda}$, is the action for a free $\beta \gamma$ system, where the conjugate momentum to $\lambda^{\alpha}$ is denoted by $\omega_{\alpha} . S_{\bar{J}}$ denotes the action for the heterotic right-moving currents and $(\bar{b}, \bar{c})$ are the right moving Virasoro ghosts. For the purpose of 
this paper, it is worth to note that the Lorentz currents $N^{a b}=\frac{1}{2} \lambda \gamma^{a b} \omega$ and ghost number current $J=\lambda^{\alpha} \omega_{\alpha}$ satisfy

$$
\begin{gathered}
N^{m n}(y) N^{p q}(z) \rightarrow \alpha^{\prime} \frac{\eta^{p[n} N^{m] q}(z)-\eta^{q[n} N^{m] p}(z)}{y-z}-3 \alpha^{2} \frac{\eta^{m[q} \eta^{p] n}}{(y-z)^{2}}, \\
J(y) J(z) \rightarrow-\frac{4}{(y-z)^{2}} .
\end{gathered}
$$

These currents have OPEs with the pure spinors

$$
N^{m n}(y) \lambda^{\alpha}(z) \rightarrow \frac{1}{2} \alpha^{\prime}\left(\gamma^{m n}\right)^{\alpha}{ }_{\beta} \frac{\lambda^{\beta}(z)}{y-z}, \quad J(y) \lambda^{\alpha}(z) \rightarrow \alpha^{\prime} \frac{\lambda^{\alpha}(z)}{y-z},
$$

while the right-moving currents satisfy

$$
\bar{J}^{I}(y) \bar{J}^{J}(z) \rightarrow \alpha^{\prime} \frac{f^{I J} \bar{J}^{K}(z)}{\bar{y}-\bar{z}}+\alpha^{\prime 2} \frac{\delta^{I J}}{(\bar{y}-\bar{z})^{2}}
$$

Physical states are defined as vertex operators in the cohomology of the BRST charge $Q=\oint d z \lambda^{\alpha} d_{\alpha}$, where $d_{\alpha}$ are the worldsheet variables corresponding to $N=1 D=10$ space-time supersymmetric derivatives.

\section{Lowest Order Constraints in $\alpha^{\prime}$}

In this section are computed the constraints coming from the nilpotency of the BRST charge and holomorphicity of the BRST current at tree level.

The action which describes the Heterotic Superstring in a curved background can be obtained by adding the massless vertex operators to the flat action and then covariantizing with respect to the $D=10 N=1$ super-reparameterization invariance [4] . The action is as follows

$$
\begin{gathered}
S=\frac{1}{2 \pi \alpha^{\prime}} \int d^{2} z\left(\frac{1}{2} \Pi^{a} \bar{\Pi}^{b} \eta_{a b}+\frac{1}{2} \Pi^{A} \bar{\Pi}^{B} B_{B A}+d_{\alpha} \bar{\Pi}^{\alpha}+\Pi^{A} \bar{J}^{I} A_{A I}+d_{\alpha} \bar{J}^{I} W_{I}^{\alpha}\right. \\
\left.\lambda^{\alpha} \omega_{\beta} \bar{J}^{I} U_{I \alpha}{ }^{\beta}+\lambda^{\alpha} \omega_{\beta} \bar{\Pi}^{C} \Omega_{C \alpha}{ }^{\beta}\right)+S_{\lambda}+S_{\bar{J}}+S_{\Phi},
\end{gathered}
$$

where $\Pi^{A}=\partial Z^{M} E_{M}^{A}(Z), \bar{\Pi}^{A}=\bar{\partial} Z^{M} E_{M}^{A}(Z)$ and $E_{M}^{A}(Z)$ is a supervielbein: $G_{M N}(Z)=$ $E_{M}^{a} E_{N}^{b} \eta_{b a} . Z^{M}$ denote the coordinates for the $D=10 N=1$ superspace $\left(X^{m}, \theta^{\mu}\right)$ with $m=0, \ldots, 9$ and $\mu=1, \ldots, 16$. $S_{\lambda}$ and $S_{\bar{J}}$, as before, are the actions for $\lambda$ and $\bar{J}^{I}=$ 
$\frac{1}{2} \mathcal{K}_{\mathcal{A B}}^{I} \bar{\psi}^{\mathcal{A}} \bar{\psi}^{\mathcal{B}}$ respectively, with $\mathcal{A}, \mathcal{B}=0, \ldots, 32 . S_{\Phi}$ is the action for the dilaton coupling to the worldsheet scalar curvature. The nilpotency of the BRST charge is guaranteed in a flat background because of the pure spinor condition. Nevertheless, when the superstring is coupled to the curved background, the background fields must be constrained in order to maintain this nilpotency [4] [13] . One can find these constrains by performing a tree level computation. To set that, one perform a background field expansion [14 by expliting every worldsheet field into a classical and quantum part, where the classical part is assumed to satisfy the classical equation of motion and the quantum part will allow to find propagators and form loops. Specifically, the following notation for the splitting will be used

$$
\begin{gathered}
Z^{M}=X_{0}^{M}+Y^{M}, \quad d_{\alpha}=d_{\alpha 0}+\widehat{d}_{\alpha}, \\
\lambda^{\alpha}=\lambda_{0}^{\alpha}+\hat{\lambda}^{\alpha}, \omega_{\alpha}=\omega_{\alpha 0}+\hat{\omega}_{\alpha}, \quad \bar{\psi}^{\mathcal{A}}=\bar{\psi}_{0}^{\mathcal{A}}+\hat{\bar{\psi}}^{\mathcal{A}} .
\end{gathered}
$$

So the expansion for the term $\frac{1}{2 \pi \alpha^{\prime}} \int d^{2} z \frac{1}{2} \partial Z^{M} \bar{\partial} Z^{N} G_{N M}$ in (3.1)in second order of the quantum fiels is

$$
\begin{gathered}
\frac{1}{2 \pi \alpha^{\prime}} \int d^{2} z\left(\frac{1}{2} \partial Y^{a} \bar{\partial} Y^{b} \eta_{a b}-\frac{1}{2} \partial Y^{a} Y^{B} \bar{\Pi}^{C} \widetilde{T}_{C B}{ }^{a}-\frac{1}{2} \bar{\partial} Y^{a} Y^{B} \Pi^{C} \widetilde{T}_{C B}{ }^{a}+\frac{1}{4} \partial Y^{B} Y^{C} \bar{\Pi}^{a} \widetilde{T}_{C B}{ }^{a}\right. \\
\left.+\frac{1}{4} \bar{\partial} Y^{B} Y^{C} \Pi^{a} \widetilde{T}_{C B}{ }^{a}+\frac{1}{2} Y^{B} Y^{C} \Pi^{D} \widetilde{T}_{D C}{ }^{a} \bar{\Pi}^{E} \widetilde{T}_{E B}{ }^{a}-\frac{1}{4} Y^{B} Y^{C} \Pi^{(a} \bar{\Pi}^{D)} \widetilde{T}_{D C B}{ }^{a}\right),
\end{gathered}
$$

where $\widetilde{T}$ is the part of the torsion which only contains derivatives of the vielbein: $\widetilde{T}_{M N}{ }^{A}=$ $\partial_{[M} E_{N]}{ }^{A}$ and $\widetilde{T}_{D C B}{ }^{A}=-\widetilde{T}_{D C}{ }^{E} \widetilde{T}_{E B}{ }^{A}+(-)^{C D} \nabla_{C} \widetilde{T}_{D B}{ }^{A}$. Repeated bosonic indices in (3.3) are assumed to be contracted with the Minkowski metric. On the other hand, the expansion for $\frac{1}{2 \pi \alpha^{\prime}} \int d^{2} z d_{\alpha} \bar{\partial} Z^{M} E_{M}{ }^{\alpha}$ is

$$
\begin{gathered}
\frac{1}{2 \pi \alpha^{\prime}} \int d^{2} z\left(\widehat{d}_{\alpha} \bar{\partial} Y^{\alpha}-\widehat{d}_{\alpha} Y^{B} \bar{\Pi}^{C} \widetilde{T}_{C B}{ }^{\alpha}+\frac{1}{2}\left(d_{\alpha 0}+\widehat{d}_{\alpha}\right) \bar{\partial} Y^{B} Y^{C} \widetilde{T}_{C B}{ }^{\alpha}\right. \\
\left.-\frac{1}{2}\left(d_{\alpha 0}+\widehat{d}_{\alpha}\right) Y^{B} \bar{\Pi}^{D} Y^{C}\left(\partial_{C} \widetilde{T}_{D B}{ }^{\alpha}+\widetilde{T}_{C D}{ }^{E} \widetilde{T}_{E B}{ }^{\alpha}\right)+\frac{1}{2} \widehat{d}_{\alpha} \bar{\Pi}^{D} Y^{M} Y^{N} \partial_{N} E_{M}{ }^{B} \widetilde{T}_{B D}{ }^{\alpha}\right)
\end{gathered}
$$

In the subsequent sections, the 0 subindex will be dropped off. The expansions for the remaining terms in the expansion of the action (3.1) are written in the appendix. From the first term in the last two expressions it can be read the propagators

$$
Y^{a}(x, \bar{x}) Y^{b}(z, \bar{z}) \rightarrow-\alpha^{\prime} \eta^{a b} \log |x-z|^{2}, \widehat{d}_{\alpha}(x) Y^{\beta}(z) \rightarrow \frac{\alpha^{\prime} \delta_{\alpha}{ }^{\beta}}{x-z} .
$$




\subsection{Nilpotency at tree level}

The propagators (3.5) allows to compute the conditions for the nilpotency of $Q_{B R S T}$ perturbatively in $\alpha^{\prime}$. In fact, one can easily compute a tree level diagram using the second propagator and the fifth term in (3.3) expanding $e^{-S}$ in a series power, giving as a result

$$
\lambda^{\alpha} d_{\alpha}(w) \lambda^{\beta} d_{\beta}(z)=\frac{1}{2} \alpha^{\prime} \frac{1}{w-z} \lambda^{\alpha} \lambda^{\beta} \Pi^{c} T_{\beta \alpha}{ }^{c}(z) .
$$

Initially one is interested in computing the tree leve diagrams coming from terms in the expansions with $\bar{\partial} Y^{A} Y^{B}$, since they will give rise to the same kind of pole as in (3.6). So, the contributions to the pole $(w-z)^{-1}$ will be

$$
\begin{gathered}
\frac{1}{2} \frac{\alpha^{\prime}}{w-z} \lambda^{\alpha} \lambda^{\beta} \Pi^{c}\left(T_{\beta \alpha}{ }^{c}+H_{\beta \alpha}^{c}\right)(z)+\frac{1}{2} \frac{\alpha^{\prime}}{w-z} \lambda^{\alpha} \lambda^{\beta} \Pi^{\gamma} H_{\gamma \beta \alpha} \\
+\frac{\alpha^{\prime}}{w-z} \lambda^{\alpha} \lambda^{\beta} d_{\gamma} T_{\beta \alpha}{ }^{\gamma}(z)+\frac{\alpha^{\prime}}{w-z} \lambda^{\alpha} \lambda^{\beta} \lambda^{\gamma} \omega_{\delta} R_{\beta \alpha \gamma}{ }^{\delta}(z) .
\end{gathered}
$$

In this notation, the Torsion superfield $T_{\beta \alpha}{ }^{\gamma}$ is given by

$$
T_{\beta \alpha}{ }^{\gamma}=\widetilde{T}_{\beta \alpha}^{\gamma}-\Omega_{\beta \alpha}^{\gamma}-\Omega_{\alpha \beta}^{\gamma}
$$

while the curvature superfield is given by

$$
R_{\alpha \beta \gamma}{ }^{\delta}=D_{\alpha} \Omega_{\beta \gamma}{ }^{\delta}+D_{\beta} \Omega_{\alpha \gamma}{ }^{\delta}+\Omega_{\alpha \gamma}{ }^{\epsilon} \Omega_{\beta \epsilon}{ }^{\delta}+\Omega_{\beta \gamma}{ }^{\epsilon} \Omega_{\alpha \epsilon}{ }^{\delta}+\widetilde{T}_{\alpha \beta}{ }^{E} \Omega_{E \gamma}{ }^{\delta},
$$

where $D_{\alpha}$ denotes the supersymmetric derivative. There are also other possible tree level contractions of $\lambda^{\alpha} d_{\alpha}(w) \lambda^{\beta} d_{\beta}(z)$ with terms including $\partial Y^{A} Y^{B}$ which will lead to

$$
\begin{gathered}
-\frac{1}{2} \alpha^{\prime} \frac{\bar{w}-\bar{z}}{(w-z)^{2}} \lambda^{\alpha} \lambda^{\beta} \bar{\Pi}^{c}\left(T_{\beta \alpha}{ }^{c}-H^{c}{ }_{\alpha \beta}\right)(z)+\frac{1}{2} \alpha^{\prime} \frac{\bar{w}-\bar{z}}{(w-z)^{2}} \lambda^{\alpha} \lambda^{\beta} \bar{\Pi}^{\gamma} H_{\gamma \alpha \beta}(z) \\
-\alpha^{\prime} \frac{\bar{w}-\bar{z}}{(w-z)^{2}} \lambda^{\alpha} \lambda^{\beta} \bar{J}^{I} F_{\alpha \beta I} .
\end{gathered}
$$

In this notation the field-strength superfield is given by

$$
F_{\alpha \beta I}=D_{\alpha} A_{\beta I}+D_{\beta} A_{\alpha I}+f_{I}^{J K} A_{\alpha J} A_{\beta K}+\widetilde{T}_{\alpha \beta}{ }^{C} A_{C I} .
$$

To compute the tree-level diagrams that give rise to the above result, one need to compute the integral

$$
\int d^{2} x \frac{1}{(w-x)(x-z)^{2}}=-\int d^{2} x \bar{\partial}_{x} \frac{(\bar{x}-\bar{w})}{x-w} \frac{1}{(x-z)^{2}}=2 \pi \frac{\bar{w}-\bar{z}}{(w-z)^{2}}
$$

From (3.7) and (3.10) it is deduced that the conditions for the nilpotency of $Q_{B R S T}$ at the lowest order in $\alpha^{\prime}$ are

$$
\lambda^{\alpha} \lambda^{\beta} T_{\alpha \beta}^{C}=0, \quad \lambda^{\alpha} \lambda^{\beta} H_{C \alpha \beta}=0, \quad \lambda^{\alpha} \lambda^{\beta} F_{\alpha \beta I}=0, \quad \lambda^{\alpha} \lambda^{\beta} \lambda^{\gamma} \omega_{\delta} R_{\beta \alpha \gamma}{ }^{\delta}=0 .
$$

These are the same set of constraints found in [4] and [13] . 


\subsection{Holomorphicity at tree level}

To compute the conditions for holomorphicity of the BRST current $\bar{\partial} j=\bar{\partial}\left(\lambda^{\alpha} d_{\alpha}\right)=0$, one must know the expansion up to first order in $Y^{\alpha}$ of the sigma model action. This expansion for the term $\frac{1}{2 \pi \alpha^{\prime}} \int d^{2} z \frac{1}{2} \partial Z^{M} \bar{\partial} Z^{N} G_{N M}$ is

$$
\frac{1}{4 \pi \alpha^{\prime}} \int d^{2}\left[\Pi^{a} \bar{\partial} Y^{b} \eta_{a b}+\bar{\Pi}^{a} \partial Y^{b} \eta_{a b}+\Pi^{b} \bar{\Pi}^{D} Y^{C} \widetilde{T}_{C D}{ }^{a} \eta_{a b}+\Pi^{D} \bar{\Pi}^{a} Y^{C} \widetilde{T}_{C D}^{b} \eta_{a b}\right]
$$

The conditions for holomorphicity will appear as conditions for vanishing to the independent couplings $\Pi^{a} \bar{\Pi}^{b}, \Pi^{\alpha} \bar{\Pi}^{b}$ and so on. For example, forming a tree level diagram

contracting $\bar{\partial} d_{\alpha}$ in $\bar{\partial} j$ with the third term in (3.14), it is obtained $\frac{1}{2} \lambda^{\alpha} \Pi^{b} \bar{\Pi}^{C} \widetilde{T}_{C \alpha}{ }^{d} \eta_{b d}$. Following this procedure with all the terms in the expansion written in the appendix up to order $Y$, it is found

$$
\begin{gathered}
\frac{1}{2} \lambda^{\alpha}\left[-\Pi^{b} \bar{\Pi}^{c}\left(T_{\alpha b}{ }^{d} \eta_{d c}+T_{\alpha c}{ }^{d} \eta_{b d}+H_{c b \alpha}\right)+\Pi^{\beta} \bar{\Pi}^{c}\left(T_{\beta \alpha b}-H_{\beta \alpha b}\right)+\Pi^{b} \bar{\Pi}^{\gamma}\left(T_{\gamma \alpha b}+H_{\gamma \alpha b}\right)\right. \\
-\Pi^{\beta} \bar{\Pi}^{\gamma} H_{\gamma \beta \alpha}-2 d_{\beta} \bar{\Pi}^{c} T_{c \alpha}{ }^{\beta}-2 d_{\beta} \bar{\Pi}^{\gamma} T_{\gamma \alpha}{ }^{\beta}+2 \Pi^{b} \bar{J}^{I} F_{b \alpha I}+2 \Pi^{\beta} \bar{J}^{I} F_{\beta \alpha I}+2 \lambda^{\beta} \omega_{\gamma} \bar{\Pi}^{d} R_{d \alpha \beta}{ }^{\gamma} \\
-2 d_{\beta} \bar{J}^{I}\left(D_{\alpha} W_{I}^{\beta}-W_{J}^{\beta} A_{\alpha K} f_{I}{ }^{J K}-U_{I \alpha}{ }^{\beta}\right)+2 \lambda^{\beta} \omega_{\gamma} \bar{J}^{I}\left(\Omega_{\alpha \delta}{ }^{\gamma} U_{I \beta}{ }^{\delta}-\Omega_{\alpha \beta}{ }^{\delta} U_{I \delta}{ }^{\gamma}+U_{J \beta}{ }^{\gamma} A_{\alpha K} f_{I} J K\right. \\
\left.\left.-W_{I}^{\delta} R_{\delta \alpha \beta} \gamma-D_{\alpha} U_{I \beta}{ }^{\gamma}\right)\right]=0 .
\end{gathered}
$$

Since $\bar{\Pi}^{\alpha}$ is related to $\bar{J}^{I}$ through $\bar{\Pi}^{\alpha}=-\bar{J}^{I} W_{I}^{\alpha}$ by using the equation of motion for the worldsheet field $d_{\alpha}$ in (3.1), one arrives at the following set of constrints for holomorphicity of the BRST current at the lowest order in $\alpha^{\prime}$

$$
\begin{gathered}
T_{\alpha(b c)}=-H_{\alpha b c}=T_{\alpha \beta}{ }^{c}-H_{\alpha \beta}{ }^{c}=T_{c \alpha}{ }^{\beta}=0, \lambda^{\alpha} \lambda^{\beta} R_{d \alpha \beta}{ }^{\gamma}=0, F_{\alpha \beta I}=-\frac{1}{2} W_{I}^{\gamma} H_{\gamma \alpha \beta}, \\
F_{\alpha b I}=-W^{\gamma} T_{\gamma \alpha b}, \nabla_{\alpha} W_{I}^{\beta}-T_{\alpha \beta}{ }^{\gamma} W_{I}^{\gamma}=U_{I \alpha}{ }^{\beta}, \lambda^{\alpha} \lambda^{\beta}\left(\nabla_{\alpha} U_{I \alpha}{ }^{\gamma}+R_{\alpha \gamma \beta}{ }^{\delta} W_{I}^{\gamma}\right)=0 .
\end{gathered}
$$

This was the same set of constraints found in [4] and [13] .

\section{Yang-Mills Chern-Simons Corrections}

In this section $\alpha^{\prime}$ corrections to the nilpotency constraints (3.13) will be computed. In the first subsection it is explained how to compute all of the twenty possible contributions to the nilpotency of the BRST charge. In the second subsection, it will be explained how, adding some counter-terms, one can find the Yang-Mills Chern-Simons 3-form. 


\subsection{One-loop Corrections to the Constraints}

In the expansion for the $\Pi^{A} \bar{J}^{I} A_{A I}$ term, the following will play a role in the computation: $\Pi^{A} Y_{0}^{B} \bar{J}_{2}^{I}\left(\partial_{B} A_{A I}+\widetilde{T}_{B A}{ }^{C} A_{C I}\right)(x)$ and $\partial Y^{A} \bar{J}_{2}^{I} A_{A I}(y)$. Contracting them with $\lambda^{\alpha} d_{\alpha}(w) \lambda^{\beta} d_{\beta}(z)$ one can form a 1-loop diagram

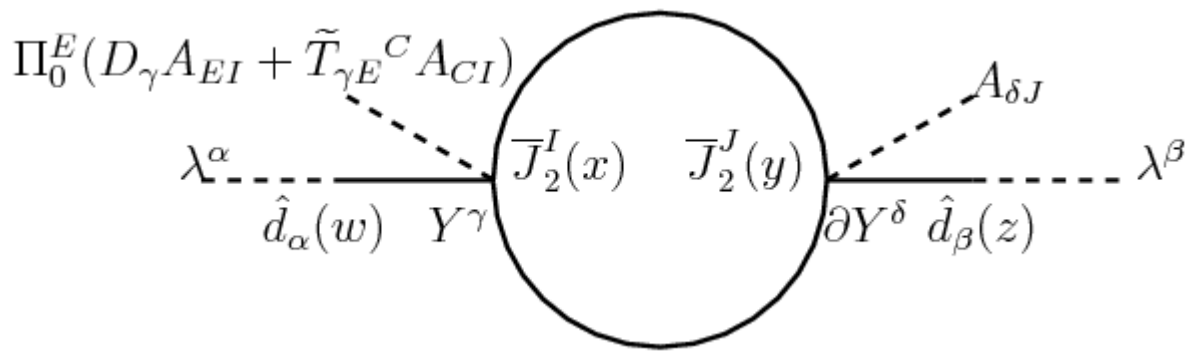

The dashed lines denote background fields while the continuous lines denote the contractions using the propagators. So one can compute how these terms contribute to the nilpotency of $Q_{B R S T}$. To determine the coefficient for this diagram, note that there is an $1 / 2$ from the expansion of $\exp [-S]$ and there is a factor of 2 coming from the possible ways to put the superfields at $x$ or $y$. Denoting the integration over the world-sheet fields by $\int[D w s f]$, it is found

$$
\begin{gathered}
\lambda^{\alpha} d_{\alpha}(w) \lambda^{\beta} d_{\beta}(z)_{I}=\frac{1}{\left(2 \pi \alpha^{\prime}\right)^{2}} \int[D w s f] \int d^{2} x d^{2} y \lambda^{\alpha} \widehat{d}_{\alpha}(w) \lambda^{\beta} \widehat{d}_{\beta}(z) \\
\Pi_{0}^{E} Y^{\gamma}\left(D_{\gamma} A_{E I}+\widetilde{T}_{\gamma E}^{F} A_{F I}\right)(x) \partial Y^{\delta} A_{\delta J}(y) \bar{J}_{2}^{I}(x) \bar{J}_{2}^{J}(y) \\
=\frac{\alpha^{\prime 2}}{(2 \pi)^{2}} \lambda^{\alpha} \lambda^{\beta} \Pi_{0}^{C} A_{\alpha I}\left(D_{\beta} A_{C I}+\widetilde{T}_{\beta C}{ }^{D} A_{D I}\right)(z) \int d^{2} x d^{2} y \frac{1}{(w-x)^{2}(z-y)} \frac{1}{(\bar{x}-\bar{y})^{2}} \\
-\frac{\alpha^{\prime 2}}{(2 \pi)^{2}} \lambda^{\alpha} \lambda^{\beta} \Pi_{0}^{C} A_{\beta I}\left(D_{\alpha} A_{C I}+\widetilde{T}_{\alpha C}{ }^{D} A_{D I}\right)(z) \int d^{2} x d^{2} y \frac{1}{(w-y)(z-x)^{2}} \frac{1}{(\bar{x}-\bar{y})^{2}}
\end{gathered}
$$

where $\bar{J}_{2}^{I}(\bar{x}) \bar{J}_{2}^{J}(\bar{y}) \rightarrow \frac{\left(\alpha^{\prime}\right)^{2} \delta^{I J}}{(\bar{x}-\bar{y})^{2}}$. The second line in the last equation is obtained from minus the first by interchanging $\alpha$ with $\beta$ and $w$ with $z$. So, just one of the integrals will be computed.

$$
\begin{gathered}
\int d^{2} x d^{2} y \frac{1}{(w-x)^{2}(z-y)(\bar{x}-\bar{y})^{2}}=\int d^{2} x d^{2} y \frac{1}{(w-x)^{2}(z-y)} \bar{\partial}_{\bar{y}} \frac{1}{\bar{x}-\bar{y}} \\
=2 \pi \int d^{2} x d^{2} y \frac{\delta^{2}(y-z)}{(w-x)^{2}(\bar{x}-\bar{y})}=2 \pi \int d^{2} x \frac{1}{(w-x)^{2}(\bar{x}-\bar{z})},
\end{gathered}
$$


where in the second step an integratetion by parts has been performed with respect to $\bar{y}$. In the last integral one can integrate by parts with respect to $x$ to obtain

$$
\int d^{2} x d^{2} y \frac{1}{(w-x)^{2}(z-y)} \frac{1}{(\bar{x}-\bar{y})^{2}}=-\frac{(2 \pi)^{2}}{w-z} .
$$

Then a first contribution to the check of nilpotency will be

$$
\lambda^{\alpha} d_{\alpha}(w) \lambda^{\beta} d_{\beta}(z)_{I}=-2 \alpha^{\prime 2} \frac{\lambda^{\alpha} \lambda^{\beta}}{w-z} \Pi_{0}^{C} A_{\beta I}\left(D_{\alpha} A_{C I}+\widetilde{T}_{\alpha C}{ }^{D} A_{D I}\right)(z)
$$

A second contribution comes from contracting $\lambda^{\alpha} d_{\alpha}(w) \lambda^{\beta} d_{\beta}(z)$ with $\partial Y^{\gamma} \bar{J}_{2}^{I} A_{\gamma I}(x) \times$ $\partial Y^{\delta} \bar{J}_{2}^{J} A_{\delta J}(y)$ as shown in the diagram.

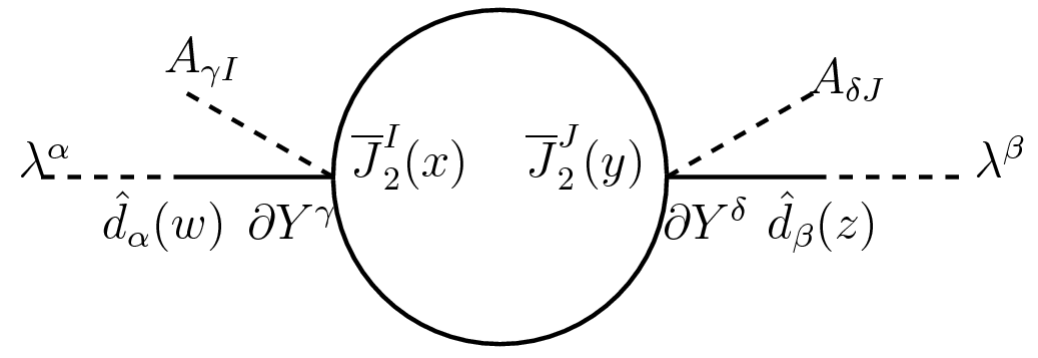

To determine the coefficient of this diagram, note that there is an $1 / 2$ coming from the Taylor expansion of $\exp (-S)$. So it is found

$\lambda^{\alpha} d_{\alpha}(w) \lambda^{\beta} d_{\beta}(z)_{I I}=\frac{\alpha^{2}}{2} \frac{\lambda^{\alpha} \lambda^{\beta}(z)}{(2 \pi)^{2}} \int d^{2} x d^{2} y\left[\frac{A_{\alpha I}(x) A_{\beta I}(y)}{(w-x)^{2}(z-y)^{2}}-\frac{A_{\beta I}(x) A_{\alpha I}(y)}{(w-y)^{2}(z-x)^{2}}\right] \frac{1}{(\bar{x}-\bar{y})^{2}}$

The second term in the integrand is obtained from minus the first by interchanging $w$ with $z$ and $\alpha$ with $\beta$. The integral left to solve is

$$
\begin{gathered}
\Gamma=\int d^{2} x d^{2} y \frac{A_{\alpha I}(x) A_{\beta I}(y)}{(w-x)^{2}(z-y)^{2}(\bar{x}-\bar{y})^{2}}=-\int d^{2} x d^{2} y \frac{\bar{\Pi}^{C} \partial_{C} A_{\alpha I}(x) A_{\beta I}(y)}{(\bar{y}-\bar{x})(w-x)^{2}(z-y)^{2}} \\
+\int d^{2} x d^{2} y \frac{A_{\alpha I}(x) A_{\beta I}(y) \partial_{x} \delta^{2}(x-w)}{(\bar{y}-\bar{x})(z-y)^{2}}
\end{gathered}
$$

where it has been integrated by parts with respect to $\bar{x}$. The first and second integral on the right hand side of (4.9) can be integrated by parts with respect to $y$ and $x$ to obtain

$\Gamma=2 \pi \int d^{2} x d^{2} y \frac{\bar{\Pi}^{C} \partial_{C} A_{\alpha I}(x) A_{\beta I}(y) \delta^{2}(y-x)}{(z-y)(w-x)^{2}}-2 \pi \int d^{2} x d^{2} y \frac{\Pi^{C} \partial_{C} A_{\alpha I}(x) A_{\beta I}(y) \delta^{2}(x-w)}{(\bar{y}-\bar{x})(z-y)^{2}}$. 
Evaluating the superfields in $z$, using (3.12)in the first integral and integrating by parts with respect to $y$ in the second, one obtains

$$
\Gamma=-(2 \pi)^{2} \frac{\bar{w}-\bar{z}}{(w-z)^{2}} \bar{\Pi}^{C} \partial_{C} A_{\alpha I} A_{\beta I}(z)-\frac{(2 \pi)^{2}}{w-z} \Pi^{C} \partial_{C} A_{\alpha I} A_{\beta I}(z) .
$$

Then

$$
\begin{gathered}
\lambda^{\alpha} d_{\alpha}(w) \lambda^{\beta} d_{\beta}(z)_{I I}=-\alpha^{2} \frac{\bar{w}-\bar{z}}{(w-z)^{2}} \lambda^{\alpha} \lambda^{\beta} \bar{\Pi}^{C} \partial_{C} A_{\alpha I} A_{\beta I}(z)-\frac{\alpha^{\prime 2}}{w-z} \lambda^{\alpha} \lambda^{\beta} \Pi^{C} \partial_{C} A_{\alpha I} A_{\beta I}(z) \\
+\alpha^{\prime 2} \frac{\bar{w}-\bar{z}}{(w-z)^{2}} \bar{\partial} \lambda^{\alpha} \lambda^{\beta} A_{\alpha I} A_{\beta I}+\frac{\alpha^{\prime 2}}{w-z} \partial \lambda^{\alpha} \lambda^{\beta} A_{\alpha I} A_{\beta I}(z)
\end{gathered}
$$

A third contribution to the nilpotency property comes from contractions of $\Pi_{0}^{A} \bar{J}_{2}^{I} A_{A I}$, twice $\partial Y^{A} \bar{J}_{2}^{I} A_{A I}$ and $\lambda^{\alpha} d_{\alpha}(w) \lambda^{\beta} d_{\beta}(z)$ giving rise to the diagram

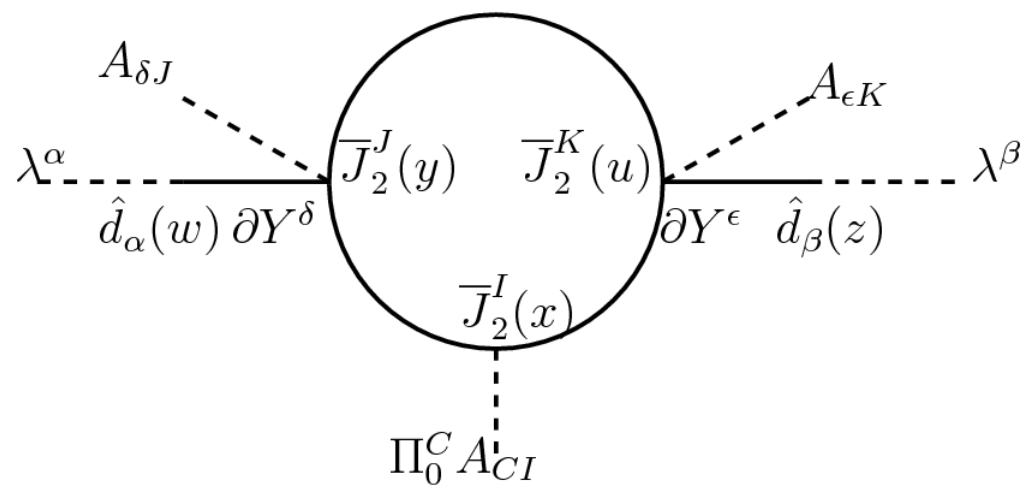

Since one is at order $S^{3}$ in the expansion of $e^{-S}$, there is an $\frac{1}{3 !}$ and also a factor of 3 from the possible ways to put the superfields at $x, y$ and $u$, so there will be a $-1 / 2$ coefficient in front:

$$
\begin{gathered}
\lambda^{\alpha} d_{\alpha}(w) \lambda^{\beta} d_{\beta}(z)_{I I I}=-\frac{1}{2\left(2 \pi \alpha^{\prime}\right)^{3}} \int[D w s f] \int d^{2} x d^{2} y d^{2} u \lambda^{\alpha} \widehat{d}_{\alpha}(w) \lambda^{\beta} \widehat{d}_{\beta}(z) \\
\Pi_{0}^{C} \bar{J}_{2}^{I} A_{C I}(x) \partial Y^{D} \bar{J}_{2}^{J} A_{D J}(y) \partial Y^{E} \bar{J}_{2}^{K} A_{E K}(u) . \\
=-\frac{1}{2(2 \pi)^{3} \alpha^{\prime}} \lambda^{\alpha} \lambda^{\beta} \Pi_{0}^{C} A_{C I} A_{\gamma J} A_{\delta K}(z) \int d^{2} x d^{2} y d^{2} u\left(\frac{\delta_{\alpha}^{\gamma} \delta_{\beta} \delta}{(w-y)^{2}(z-u)^{2}}\right.
\end{gathered}
$$




$$
\left.-\frac{\delta_{\alpha}{ }^{\delta} \delta_{\beta} \gamma}{(w-u)^{2}(z-y)^{2}}\right) \bar{J}_{2}^{I}(x) \bar{J}_{2}^{J}(y) \bar{J}_{2}^{K}(u) .
$$

It is not hard to verify that

$$
\bar{J}_{2}^{I}(x) \bar{J}_{2}^{J}(y) \bar{J}_{2}^{K}(u)=\frac{\left(\alpha^{\prime}\right)^{3} f^{I J K}}{(\bar{x}-\bar{y})(\bar{y}-\bar{u})(\bar{x}-\bar{u})}+\ldots,
$$

where by ... is meant less singular poles which are not important in this computation. Then the type of integrals that must be computed are

$$
\Gamma_{1}=\int d^{2} x d^{2} y d^{2} u \frac{1}{(w-y)^{2}(z-u)^{2}(\bar{x}-\bar{y})(\bar{y}-\bar{u})(\bar{x}-\bar{u})} .
$$

The integral in $x$ gives

$$
\int d^{2} x \frac{1}{(\bar{x}-\bar{y})(\bar{x}-\bar{u})}=\int d^{2} x \partial_{x}\left(\frac{x-y}{\bar{x}-\bar{y}}\right) \frac{1}{\bar{x}-\bar{u}}=-2 \pi \frac{y-u}{\bar{y}-\bar{u}},
$$

so 4.17 ) yields

$$
\Gamma_{1}=-2 \pi \int d^{2} y d^{2} u \partial_{y}\left(\frac{1}{w-y}\right) \frac{y-u}{(z-u)^{2}(\bar{y}-\bar{u})^{2}} .
$$

Integrating by parts in $y, \bar{y}$ and then in $u$ it is found $\Gamma_{1}=(2 \pi)^{3} /(w-z)$. In this way (4.8) gives

$$
\lambda^{\alpha} d_{\alpha}(w) \lambda^{\beta} d_{\beta}(z)_{I I I}=-\left(\alpha^{\prime}\right)^{2} \frac{\lambda^{\alpha} \lambda^{\beta}}{w-z} f^{I J K} \Pi_{0}^{C} A_{C I} A_{\alpha J} A_{\beta K}(z)
$$

Note that a fourth loop could be formed with $\frac{1}{4} \bar{\partial} Y^{\alpha} Y^{\beta} \Pi^{c}\left(T_{\beta \alpha}{ }^{c}+H^{c}{ }_{\beta \alpha}\right), \widehat{d}_{\alpha} \bar{J}_{2}^{I} W_{I}^{\alpha}$ and $\partial Y^{\alpha} \bar{J}_{2}^{I} A_{\alpha I}$ as shown in the diagram below.

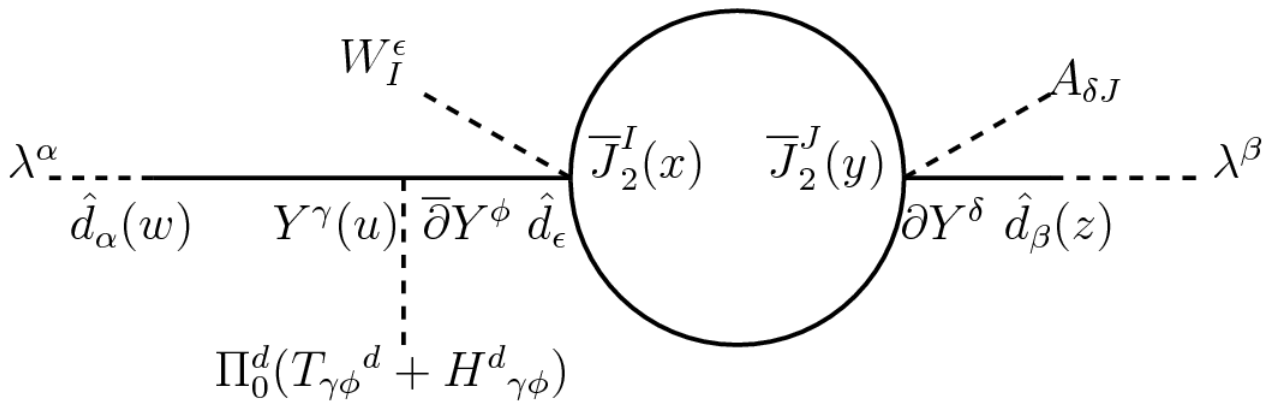

In this case, one is also at the order $S^{3}$, so there is an $\frac{1}{3 !}$ which is cancelled by the symmetry factor responsible for the localization of the superfields, either at $x, y$ or $u$. The $\frac{1}{4}$ coming from the coefficient of the term with $\Pi^{c}$ is cancelled by a symmetry factor of the possible ways of contraction: 


$$
\begin{gathered}
\lambda^{\alpha} d_{\alpha}(w) \lambda^{\beta} d_{\beta}(z)_{I V}=-\frac{\alpha^{\prime 2}}{(2 \pi)^{2}} \lambda^{\alpha} \lambda^{\beta} \Pi^{c}\left(T_{\delta \alpha}{ }^{c}+H^{c}{ }_{\delta \alpha}\right) W_{I}^{\delta} A_{\beta I}(z) \times \\
\int d^{2} x d^{2} y d^{2} u \frac{\delta^{2}(x-w)}{(z-u)^{2}(y-x)(\bar{y}-\bar{u})^{2}}
\end{gathered}
$$

Integrating $x$ one has to solve

$$
\int d^{2} y d^{2} u \frac{1}{(z-u)^{2}(y-w)(\bar{y}-\bar{u})^{2}}=-2 \pi \int d^{2} y d^{2} u \frac{\delta^{2}(y-w)}{(\bar{u}-\bar{y})(z-u)^{2}}=-\frac{(2 \pi)^{2}}{w-z} .
$$

Then

$$
\lambda^{\alpha} d_{\alpha}(w) \lambda^{\beta} d_{\beta}(z)_{I V}=\frac{\alpha^{\prime 2}}{w-z} \lambda^{\alpha} \lambda^{\beta} \Pi^{c}\left(T_{\alpha \delta}^{c}+H_{\alpha \delta}^{c}\right) W_{I}^{\delta} A_{\beta I}(z)
$$

Considering the same last diagram but with the vertex $\frac{1}{4} \Pi^{\gamma} H_{\gamma \beta \alpha}$ instead of $\frac{1}{4} \Pi^{c}\left(T_{\beta \alpha}{ }^{c}+H_{\beta \alpha}{ }^{c}\right)$, gives a fifth contribution to the coupling to $\Pi^{\gamma}$

$$
\lambda^{\alpha} d_{\alpha}(w) \lambda^{\beta} d_{\beta}(z)_{V}=\frac{\alpha^{2}}{w-z} \lambda^{\alpha} \lambda^{\beta} \Pi^{\gamma} H_{\gamma \alpha \delta} W_{I}^{\delta} A_{\beta I}(z)
$$

A sixth contribution can be formed with $\frac{1}{4} \Pi^{c} \bar{\partial} Y^{A} Y^{B}\left(\widetilde{T}_{B A}{ }^{c}+H^{c}{ }_{B A}\right)$ and twice $\partial Y^{A} \bar{J}_{2}^{I} A_{A I}$ :

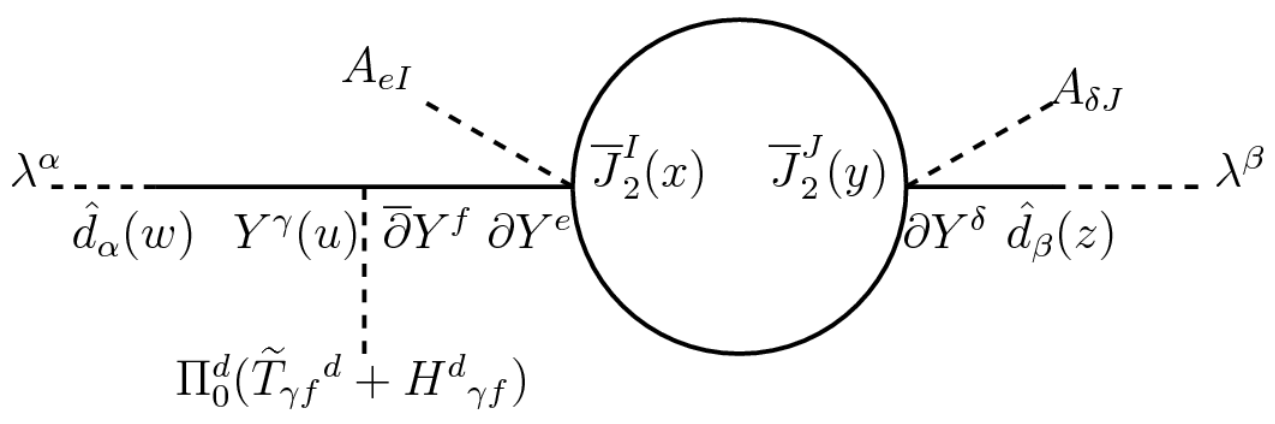

There are 8 possible ways of making the contractions, a 3 factor from the possible ways to put the superfields at $x, y$ or $u$, an $1 / 3$ ! because one is at $S^{3}$ in the expansion, and the factor of $1 / 4$ of the $\Pi^{c}$ term gives a one coefficient:

$$
\begin{gathered}
\lambda^{\alpha} d_{\alpha}(w) \lambda^{\beta} d_{\beta}(z)_{V I}=-\frac{\alpha^{\prime 2}}{(2 \pi)^{2}} \lambda^{\alpha} \lambda^{\beta} \Pi^{c}\left(\widetilde{T}_{d \alpha}{ }^{c}+H_{d \alpha}^{c}\right) A_{d I} A_{\beta I}(z) \times \\
\int d^{2} x d^{2} y d^{2} u \frac{\delta^{2}(x-w)}{(y-x)(z-u)^{2}} \frac{1}{(\bar{y}-\bar{u})^{2}} .
\end{gathered}
$$


The integral is the same as in (4.22), so the answer is

$$
\lambda^{\alpha} d_{\alpha}(w) \lambda^{\beta} d_{\beta}(z)_{V I}=\frac{\alpha^{\prime 2}}{w-z} \lambda^{\alpha} \lambda^{\beta} \Pi^{c}\left(\widetilde{T}_{d \alpha}{ }^{c}+H_{d \alpha}^{c}\right) A_{d I} A_{\beta I}(z)
$$

In the same way, the last diagram but with the vertex $\frac{1}{4} \Pi^{\gamma} H_{\gamma B A}$ instead of $\frac{1}{4} \Pi^{c}\left(T_{B A^{c}}+H_{B A^{c}}\right)$ leads to a seventh contribution

$$
\lambda^{\alpha} d_{\alpha}(w) \lambda^{\beta} d_{\beta}(z)_{V I I}=\frac{\alpha^{2}}{w-z} \lambda^{\alpha} \lambda^{\beta} \Pi^{\gamma} H_{\gamma d \alpha} A_{d I} A_{\beta I}(z)
$$

An eight contribution can be formed with $-\frac{1}{2} \bar{\partial} Y^{a} Y^{\beta} \Pi^{C} \widetilde{T}_{C \beta}{ }^{a}$ and twice $\partial Y^{A} \bar{J}_{2}^{I} A_{A I}$ :

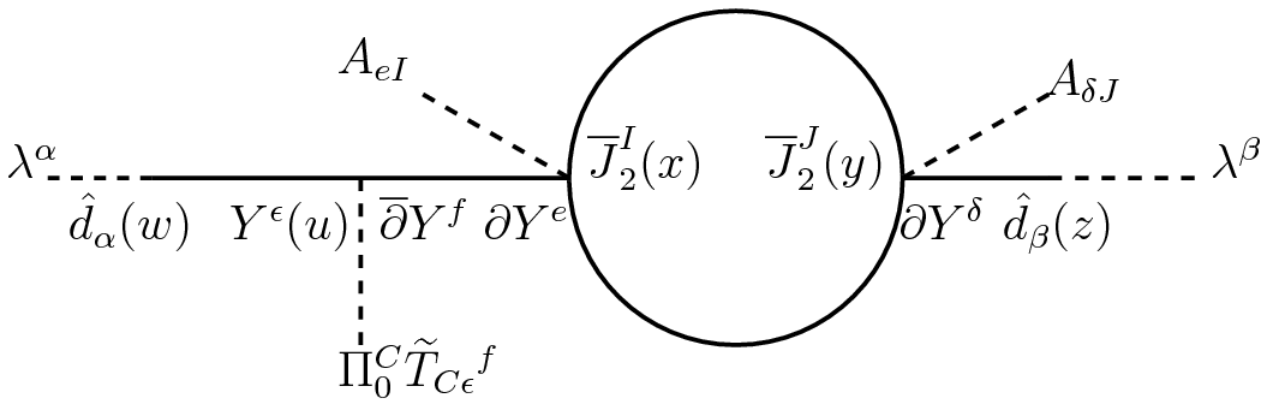

There are 4 possible ways of making the contractions, a 3 factor from the possible ways to put the superfields at $x, y$ or $u$, an $\frac{1}{3 !}$ because one is at $S^{3}$ order in the expansion and a factor of $1 / 2$ of the $\Pi^{a}$ coefficient, giving at the end a 1 coefficient:

$$
\begin{gathered}
\lambda^{\alpha} d_{\alpha}(w) \lambda^{\beta} d_{\beta}(z)_{V I I I}=-\frac{\alpha^{\prime 2}}{(2 \pi)^{3}} \lambda^{\alpha} \lambda^{\beta} \Pi^{C} \widetilde{T}_{C \alpha}{ }^{d} A_{\beta I} A_{d I}(z) \times \\
\int d^{2} x d^{2} y d^{2} u \frac{-2 \pi \delta^{2}(u-x)}{(w-x)(z-y)^{2}} \frac{1}{(\bar{u}-\bar{y})^{2}} .
\end{gathered}
$$

Integrating in $u$, the integral one has to solve is

$$
\int d^{2} x d^{2} y \frac{1}{(w-x)(z-y)^{2}(\bar{x}-\bar{y})^{2}}=2 \pi \int d^{2} x d^{2} y \frac{\delta^{2}(x-w)}{(z-y)^{2}(\bar{y}-\bar{x})}=\frac{(2 \pi)^{2}}{w-z}
$$

then

$$
\lambda^{\alpha} d_{\alpha}(w) \lambda^{\beta} d_{\beta}(z)_{V I I I}=\frac{\alpha^{2}}{w-z} \lambda^{\alpha} \lambda^{\beta} \Pi^{C} \widetilde{T}_{C \alpha}{ }^{d} A_{\beta I} A_{d I}(z)
$$

Let's consider the couplings to $\bar{\Pi}^{A}$. 
A diagram like (4.21) can be formed with $\frac{1}{4} \bar{\Pi}^{c} \partial Y^{A} Y^{B}\left(\widetilde{T}_{B A^{c}}-H^{c}{ }_{B A}\right), \partial Y^{A} \bar{J}_{2}^{I} A_{A I}$ and $\widehat{d}_{\alpha} \bar{J}_{2}^{I} W_{I}^{\alpha}$. There are 4 possible ways of making the contractions, a 6 factor from the possible ways to put the superfields at $x, y$ or $u$, an $\frac{1}{3 !}$ because one is at $S^{3}$ order in the expansion and a factor of $1 / 4$ of the $\bar{\Pi}^{c}$ coefficient, giving at the end a 1 coefficient to this ninth contribution:

$$
\begin{gathered}
\lambda^{\alpha} d_{\alpha}(w) \lambda^{\beta} d_{\beta}(z)_{I X}=\frac{\alpha^{\prime 2}}{(2 \pi)^{3}} \lambda^{\alpha} \lambda^{\beta} \bar{\Pi}^{c}\left(T_{\delta \alpha}{ }^{c}-H_{\delta \alpha}^{c}\right) W_{I}^{\delta} A_{\beta I}(z) \times \\
\int d^{2} x d^{2} y d^{2} u \frac{1}{(w-x)^{2}(z-u)^{2}(y-x)(\bar{y}-\bar{u})^{2}}
\end{gathered}
$$

Integrating $\bar{y}$ by parts, one is left to solve the integral

$$
\int d^{2} x d^{2} y d^{2} u \frac{\delta^{2}(y-x)}{(w-x)^{2}(z-u)^{2}(\bar{u}-\bar{y})}=2 \pi \int d^{2} x \frac{1}{(w-x)(z-x)^{2}} .
$$

The right hand side in the last equation is the same as (3.12), so

$$
\lambda^{\alpha} d_{\alpha}(w) \lambda^{\beta} d_{\beta}(z)_{I X}=-\alpha^{\prime 2} \frac{\bar{w}-\bar{z}}{(w-z)^{2}} \lambda^{\alpha} \lambda^{\beta} \bar{\Pi}^{c}\left(T_{\delta \alpha}{ }^{c}-H_{\delta \alpha}^{c}\right) W_{I}^{\delta} A_{\beta I}(z) .
$$

In the same way, considering vertex $-\frac{1}{4} \bar{\Pi}^{\gamma} H_{\gamma B A}$ instead of $-\frac{1}{4} \bar{\Pi}^{c}\left(\widetilde{T}_{B A}^{c}-H_{B A}{ }^{c}\right)$ leads to the tenth contribution

$$
\lambda^{\alpha} d_{\alpha}(w) \lambda^{\beta} d_{\beta}(z)_{X}=\alpha^{\prime 2} \frac{\bar{w}-\bar{z}}{(w-z)^{2}} \lambda^{\alpha} \lambda^{\beta} \bar{\Pi}^{\gamma} H_{\gamma \delta \alpha} W_{I}^{\delta} A_{\beta I}(z)
$$

An eleventh contribution comes from a diagram like (4.26) which can be formed with $\frac{1}{4} \bar{\Pi}^{c} \partial Y^{A} Y^{B}\left(\widetilde{T}_{B A}{ }^{c}-H^{c}{ }_{B A}\right)$ and twice $\partial Y^{A} \partial \bar{J}_{2}^{I} A_{A I}$. There are 8 possible ways of making the contractions, a 3 factor from the possible ways to put the superfields at $x, y$ or $u$, an $\frac{1}{3 !}$ because one is at $S^{3}$ order in the expansion and a factor of $1 / 4$ of the $\bar{\Pi}^{c}$ coefficient, giving at the end $\mathrm{a}+$ coefficient:

$$
\begin{gathered}
\lambda^{\alpha} d_{\alpha}(w) \lambda^{\beta} d_{\beta}(z)_{X I}=\frac{\alpha^{\prime 2}}{(2 \pi)^{3}} \lambda^{\alpha} \lambda^{\beta} \bar{\Pi}^{c}\left(\widetilde{T}_{d \alpha}{ }^{c}-H_{d \alpha}^{c}\right) A_{d I} A_{\beta I}(z) \times \\
\int d^{2} x d^{2} y d^{2} u \frac{1}{(w-x)^{2}(z-u)^{2}(y-x)(\bar{u}-\bar{y})} .
\end{gathered}
$$

The last integral is the same as the integral in (4.34), so the result is

$$
\lambda^{\alpha} d_{\alpha}(w) \lambda^{\beta} d_{\beta}(z)_{X I}=-\alpha^{\prime 2} \frac{\bar{w}-\bar{z}}{(w-z)^{2}} \lambda^{\alpha} \lambda^{\beta} \bar{\Pi}^{c}\left(\widetilde{T}_{d \alpha}{ }^{c}-H_{d \alpha}^{c}\right) A_{d I} A_{\beta I}(z) .
$$


In the same way, a twelfth contribution comes from considering the vertex $-\frac{1}{4} \bar{\Pi}^{\gamma} H_{\gamma B A}$ instead of the vertex $\frac{1}{4} \bar{\Pi}^{c}\left(\widetilde{T}_{B A^{c}}-H_{B A}{ }^{c}\right)$, leading to

$$
\lambda^{\alpha} d_{\alpha}(w) \lambda^{\beta} d_{\beta}(z)_{X I I}=\alpha^{2} \frac{\bar{w}-\bar{z}}{(w-z)^{2}} \lambda^{\alpha} \lambda^{\beta} \bar{\Pi}^{\gamma} H_{\gamma d \alpha} A_{d I} A_{\beta I}(z) .
$$

Another diagram like (4.30) can be formed with $-\frac{1}{2} \partial Y^{a} Y^{\beta} \bar{\Pi}^{C} \widetilde{T}_{C \beta}{ }^{a}, \partial Y^{a} \bar{J}_{2}^{I} A_{a I}$ and $\partial Y^{\alpha} \bar{J}_{2}^{I} A_{\alpha I}$, giving rise to a thirteenth contribution

$$
\lambda^{\alpha} d_{\alpha}(w) \lambda^{\beta} d_{\beta}(z)_{X I I I}=-\alpha^{\prime 2} \frac{\bar{w}-\bar{z}}{(w-z)^{2}} \lambda^{\alpha} \lambda^{\beta} \bar{\Pi}^{C} \widetilde{T}_{C \alpha}{ }^{d} A_{d I} A_{\beta I}(z)
$$

A fourteenth contribution and the last for the couplings to $\bar{\Pi}^{A}$ can be formed with $-\widehat{d}_{\alpha} Y^{B} \bar{\Pi}^{C} \widetilde{T}_{C B}{ }^{\alpha}$ and twice $\partial Y^{A} \bar{J} A_{A I}$ :

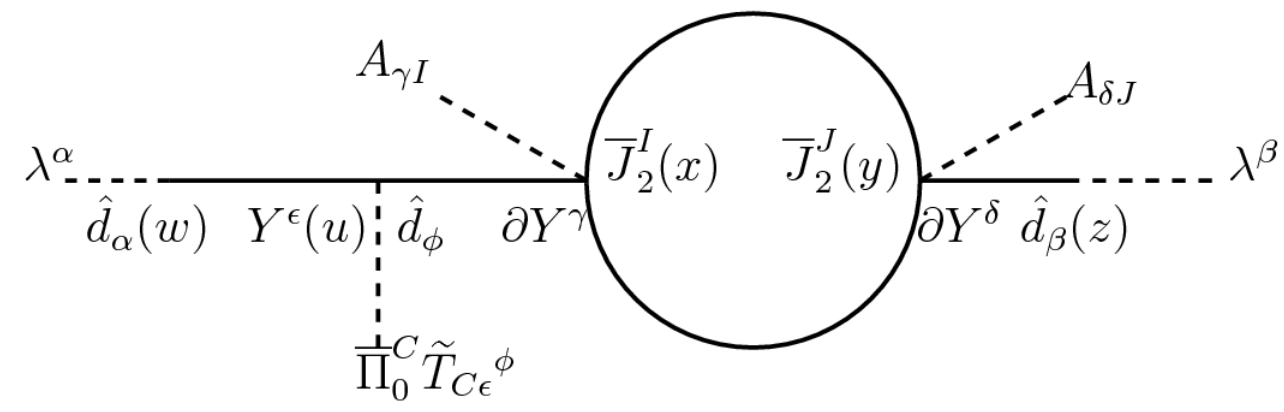

giving as result

$$
\lambda^{\alpha} d_{\alpha}(w) \lambda^{\beta} d_{\beta}(z)_{X I V}=2 \alpha^{\prime} \frac{\bar{w}-\bar{z}}{(w-z)^{2}} \lambda^{\alpha} \lambda^{\beta} \bar{\Pi}^{C} A_{\beta I} \widetilde{T}_{C \alpha}^{\gamma} A_{\gamma I}
$$

Let's consider the couplings to $\bar{J}_{0}^{I}$

A fifteenth contribution to the nilpotency will come from a diagram formed with $\frac{1}{2} \partial Y^{A} Y^{B} \bar{J}_{0}^{I}\left(\partial_{[B} A_{A] I}+\widetilde{T}_{B A}^{C} A_{C I}\right), \widehat{d}_{\alpha} \bar{J}_{2}^{I} W_{I}^{\alpha}$ and $\partial Y^{\alpha} \bar{J}_{2}^{I} A_{\alpha I}$ :

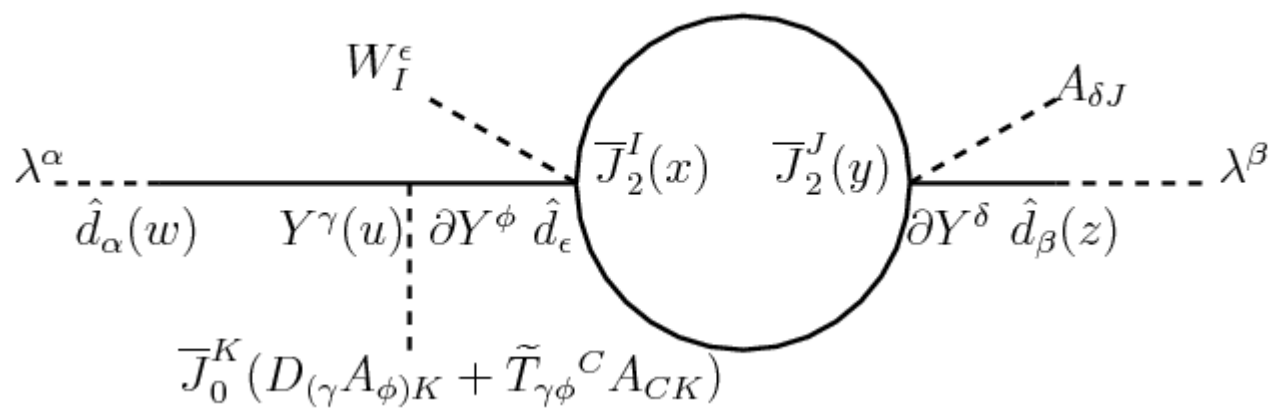


There are 4 possible ways of making the contractions, a 6 factor from the possible ways to put the superfields at $x, y$ or $u$, an $\frac{1}{3 !}$ because one is at the $S^{3}$ order in the expansion and a factor of $1 / 2$ of the $\bar{J}_{0}^{I}$ coefficient, giving at the end a 2 factor:

$$
\begin{gathered}
\lambda^{\alpha} d_{\alpha}(w) \lambda^{\beta} d_{\beta}(z)_{X V}=\frac{2 \alpha^{\prime 2}}{(2 \pi)^{3}} \lambda^{\alpha} \lambda^{\beta} \bar{J}_{0}^{I}\left(D_{(\gamma} A_{\alpha) I}+\widetilde{T}_{\gamma \alpha}{ }^{C} A_{C I}\right) W_{J}^{\gamma} A_{\beta J}(z) \times \\
\int d^{2} x d^{2} y d^{2} u \frac{1}{(w-x)^{2}(z-u)^{2}(y-x)(\bar{u}-\bar{y})^{2}} .
\end{gathered}
$$

The last integral is again the same as in (4.34), so the result is

$$
\lambda^{\alpha} d_{\alpha}(w) \lambda^{\beta} d_{\beta}(z)_{X V}=-2 \alpha^{\prime 2} \frac{\bar{w}-\bar{z}}{(w-z)^{2}} \lambda^{\alpha} \lambda^{\beta} \bar{J}_{0}^{I}\left(D_{(\gamma} A_{\alpha) I}+\widetilde{T}_{\gamma \alpha}{ }^{C} A_{C I}\right) W_{J}^{\gamma} A_{\beta J}(z) .
$$

A sixteenth contribution can be formed with $\frac{1}{2} \partial Y^{A} Y^{B} \bar{J}_{0}^{I}\left(\partial_{[B} A_{A] I}+\widetilde{T}_{B A}{ }^{C} A_{C I}\right)$ and twice $\partial Y^{A} \bar{J}_{2}^{I} A_{A I}$ :

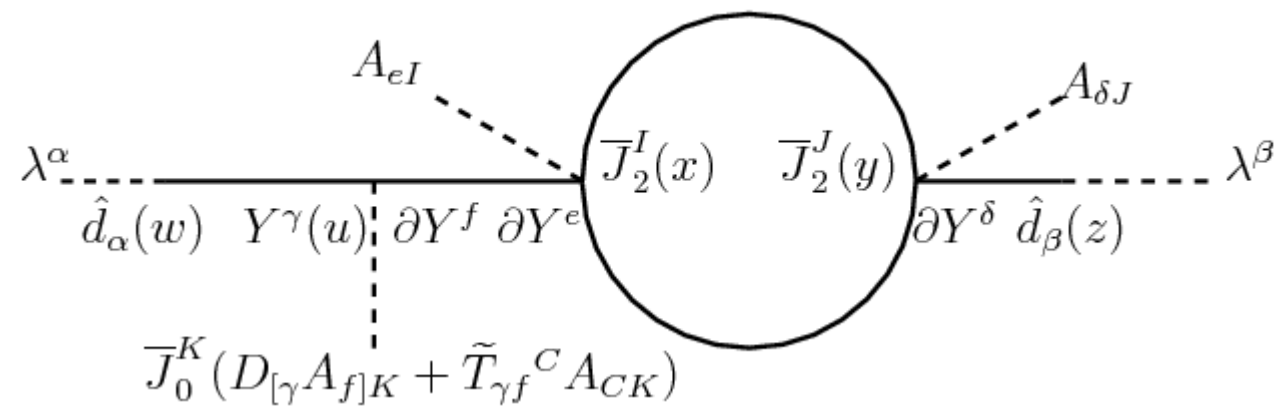

There are 8 possible ways of making the contractions, a 3 factor from the possible ways to put the superfields at $x, y$ or $u$, an $\frac{1}{3 !}$ because one is at the $S^{3}$ order in the expansion and a factor of $1 / 2$ of the $\bar{J}_{0}^{I}$ coefficient, giving at the end a 2 coefficient:

$$
\begin{gathered}
\lambda^{\alpha} d_{\alpha}(w) \lambda^{\beta} d_{\beta}(z)_{X V I}=2 \frac{\alpha^{\prime 2}}{(2 \pi)^{3}} \lambda^{\alpha} \lambda^{\beta} \bar{J}_{0}^{I}\left(\partial_{[c} A_{\alpha] I}+\widetilde{T}_{c \alpha}{ }^{D} A_{D I}\right) A_{c J} A_{\beta J}(z) \times \\
\int d^{2} x d^{2} y d^{2} u \frac{1}{(w-x)^{2}(z-u)^{2}(y-x)(\bar{y}-\bar{u})^{2}},
\end{gathered}
$$

which contains the same integral as before, so the result is

$$
\lambda^{\alpha} d_{\alpha}(w) \lambda^{\beta} d_{\beta}(z)_{X V I}=-2 \alpha^{\prime 2} \frac{\bar{w}-\bar{z}}{(w-z)^{2}} \lambda^{\alpha} \lambda^{\beta} \bar{J}_{0}^{I}\left(\partial_{[c} A_{\alpha] I}+\widetilde{T}_{c \alpha}{ }^{D} A_{D I}\right) A_{c J} A_{\beta J}(z)
$$

Finally, let's consider the couplings to $d_{\alpha}$. 
A seventeenth contribution can be formed with $\frac{1}{2} d_{\alpha} \bar{\partial} Y^{\beta} Y^{\gamma} \widetilde{T}_{\gamma \beta}^{\alpha}, \widehat{d}_{\alpha} \bar{J}_{2}^{I} W_{I}^{\alpha}$ and $\partial Y^{\alpha} \bar{J}_{2}^{I} A_{\alpha I}$ :

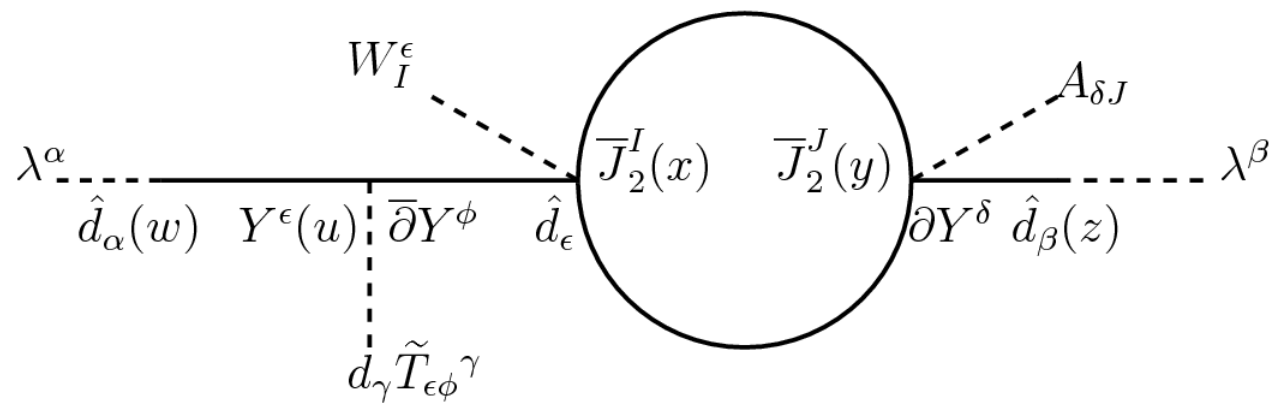

There are 4 possible ways of making the contractions, a 6 factor from the possible ways to put the superfields at $x, y$ or $u$, an $\frac{1}{3 !}$ because one is at the $S^{3}$ order in the expansion and a factor of $1 / 2$ of the $d_{\alpha}$ coefficient, giving at the end a 2 coefficient:

$$
\begin{gathered}
\lambda^{\alpha} d_{\alpha}(w) \lambda^{\beta} d_{\beta}(z)_{X V I I}=-2 \frac{\alpha^{\prime 2}}{(2 \pi)^{2}} \lambda^{\alpha} \lambda^{\beta} d_{\gamma} \widetilde{T}_{\delta \alpha}{ }^{\gamma} W_{I}^{\delta} A_{\beta I}(z) \times \\
\int d^{2} x d^{2} y d^{2} u \frac{\delta^{2}(x-w)}{(z-u)^{2}(y-x)(\bar{y}-\bar{u})^{2}}
\end{gathered}
$$

Integrating $x$, the integral that is left to solve is

$$
\int d^{2} y d^{2} u \frac{1}{(z-u)^{2}(y-w)(\bar{y}-\bar{u})^{2}}=-2 \pi \int d^{2} y d^{2} u \frac{\delta^{2}(y-w)}{(\bar{u}-\bar{y})(z-u)^{2}}=-\frac{(2 \pi)^{2}}{w-z}
$$

So,

$$
\lambda^{\alpha} d_{\alpha}(w) \lambda^{\beta} d_{\beta}(z)_{X V I I}=\frac{2 \alpha^{\prime 2}}{w-z} \lambda^{\alpha} \lambda^{\beta} d_{\gamma} \widetilde{T}_{\delta \alpha}^{\gamma} W_{I}^{\delta} A_{\beta I}(z)
$$

An eighteenth contribution can be formed with $\frac{1}{2} d_{\alpha} \bar{\partial} Y^{B} Y^{C} \widetilde{T}_{C B}{ }^{\alpha}$ and twice $\partial Y^{A} \bar{J}_{2}^{I} A_{A I}$ :

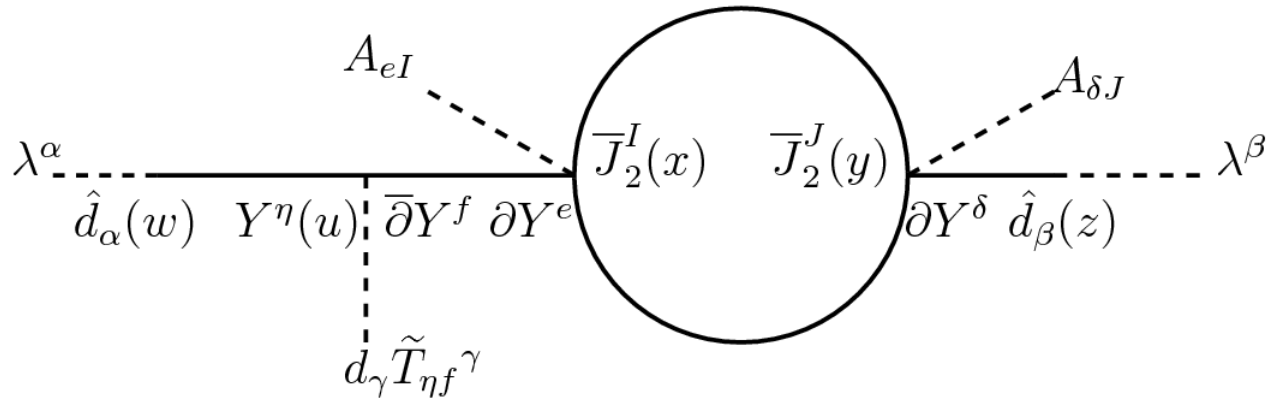

There are 8 possible ways of making the contractions, a 3 factor from the possible ways to put the superfields at $x, y$ and $u$, an $\frac{1}{3 !}$ because one is at the $S^{3}$ order in the expansion and a factor of $1 / 2$ of the $d_{\alpha}$ coefficient, giving a 2 coefficient: 


$$
\begin{gathered}
\lambda^{\alpha} d_{\alpha}(w) \lambda^{\beta} d_{\beta}(z)_{X V I I I}=2 \frac{\alpha^{\prime 2}}{(2 \pi)^{2}} \lambda^{\alpha} \lambda^{\beta} d_{\gamma} \widetilde{T}_{c \alpha}{ }^{\gamma} A_{c I} A_{\beta I}(z) \times \\
\int d^{2} x d^{2} y d^{2} u \frac{\delta^{2}(x-w)}{(z-u)^{2}(y-x)(\bar{y}-\bar{u})^{2}} .
\end{gathered}
$$

This integral is the same as in (4.52), so the result is

$$
\lambda^{\alpha} d_{\alpha}(w) \lambda^{\beta} d_{\beta}(z)_{X V I I I}=-\frac{2 \alpha^{\prime 2}}{w-z} \lambda^{\alpha} \lambda^{\beta} d_{\gamma} \widetilde{T}_{c \alpha}{ }^{\gamma} A_{c I} A_{\beta I}(z) .
$$

Because of the pure spinor condition, the action is invariant under $\delta \omega_{\alpha}=\left(\Lambda_{b} \gamma^{b} \lambda\right)_{\alpha}$, so $U_{I \alpha}{ }^{\beta}=U_{I} \delta_{\alpha}^{\beta}+\frac{1}{4} U_{I c d}\left(\gamma^{c d}\right)_{\alpha}{ }^{\beta}$. It can be formed a nineteenth one-loop diagram by contracting $J \bar{J}_{2}^{I} U_{I}(x)$ with $\partial Y^{\alpha} \bar{J}_{2}^{I} A_{\alpha I}$ :

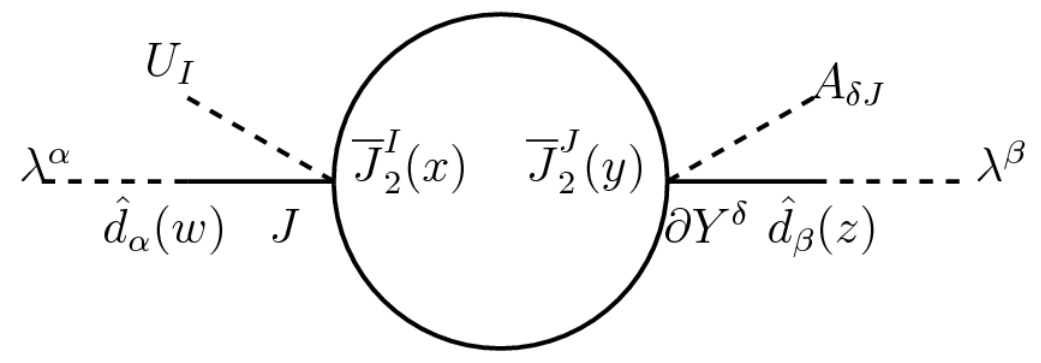

giving the contribution

$$
\lambda^{\alpha} d_{\alpha}(w) \lambda^{\beta} d_{\beta}(z)_{X I X}=-2 \frac{\alpha^{\prime 2}}{w-z} \lambda^{\alpha} \lambda^{\beta} d_{\gamma} \delta_{\alpha}^{\gamma} A_{\beta I} U_{I}
$$

Similarly, a diagram like (4.57) can be formed contracting $\frac{1}{2} N^{a b} \bar{J}_{2}^{I} U_{I a b}(x)$ with $\partial Y^{\alpha} \bar{J}_{2}^{I} A_{\alpha I}$, giving as contribution

$$
\lambda^{\alpha} d_{\alpha}(w) \lambda^{\beta} d_{\beta}(z)_{X X}=-\frac{1}{2} \frac{\alpha^{\prime 2}}{w-z} \lambda^{\alpha} \lambda^{\beta} d_{\gamma}\left(\gamma^{e f}\right)_{\alpha}^{\gamma} U_{\text {Ief }} A_{\beta I}
$$

Now, the results will be summarized by adding up the twenty one-loop contributions to the tree level constraints. Each independent worldsheet coupling will receive corrections, as indicated below:

Corrections to the the coupling to $\Pi^{c}$

$$
\begin{aligned}
& \frac{1}{2} \frac{\alpha^{\prime}}{w-z} \lambda^{\alpha} \lambda^{\beta} \Pi^{c}\left[\left(T_{\beta \alpha}{ }^{c}+H^{c}{ }_{\beta \alpha}\right)-4 \alpha^{\prime} A_{\beta I}\left(D_{\alpha} A_{c I}+\widetilde{T}_{\alpha c}{ }^{D} A_{D I}\right)+2 \alpha^{\prime} A_{\beta I} \partial_{c} A_{\alpha I}\right. \\
&\left.-2 \alpha^{\prime} f^{I J K} A_{c I} A_{\alpha J} A_{\beta K}+2 \alpha^{\prime}\left(T_{\alpha \delta}{ }^{c}+H^{c}{ }_{\alpha \delta}\right) W_{I}^{\delta} A_{\beta I}+2 \alpha^{\prime}\left(T_{d \alpha}{ }^{c}+T_{c \alpha}{ }^{e} \eta_{e d}+H^{c}{ }_{d \alpha}\right) A_{d I} A_{\beta I}\right](z) .
\end{aligned}
$$


Corrections to the coupling to $\bar{\Pi}^{c}$

$$
\begin{gathered}
-\frac{1}{2} \alpha^{\prime} \frac{\bar{w}-\bar{z}}{(w-z)^{2}} \lambda^{\alpha} \lambda^{\beta} \bar{\Pi}^{c}\left[\left(T_{\beta \alpha}{ }^{c}-H_{\alpha \beta}^{c}\right)-2 \alpha^{\prime} A_{\beta I} \partial_{c} A_{\alpha I}+2 \alpha^{\prime}\left(T_{\delta \alpha}{ }^{c}-H^{c}{ }_{\delta \alpha}\right) W_{I}^{\delta} A_{\beta I}\right. \\
\left.+2 \alpha^{\prime}\left(T_{d \alpha}{ }^{c}+T_{c \alpha}{ }^{e} \eta_{e d}-H_{d \alpha}^{c}\right) A_{d I} A_{\beta I}-4 \alpha^{\prime} A_{\beta I} \widetilde{T}_{c \alpha}{ }^{\gamma} A_{\gamma I}\right](z)
\end{gathered}
$$

Corrections to the coupling to $\Pi^{\gamma}$

$$
\begin{aligned}
& \frac{1}{2} \frac{\alpha^{\prime}}{w-z} \lambda^{\alpha} \lambda^{\beta} \Pi^{\gamma}\left[H_{\gamma \beta \alpha}-4 \alpha^{\prime} A_{\beta I}\left(D_{\alpha} A_{\gamma I}+\widetilde{T}_{\alpha \gamma}{ }^{D} A_{D I}\right)-2 \alpha^{\prime} A_{\beta I} D_{\gamma} A_{\alpha I}\right. \\
& \left.-2 \alpha^{\prime} f^{I J K} A_{\gamma I} A_{\alpha J} A_{\beta K}+2 \alpha^{\prime} H_{\gamma \alpha \delta} W_{I}^{\delta} A_{\beta I}+2 \alpha^{\prime}\left(T_{\gamma \alpha d}-H_{\gamma \alpha d}\right) A_{d I} A_{\beta I}\right](z) .
\end{aligned}
$$

Corrections to the coupling to $\bar{\Pi}^{\gamma}$

$$
\begin{gathered}
\frac{1}{2} \alpha^{\prime} \frac{\bar{w}-\bar{z}}{(w-z)^{2}} \lambda^{\alpha} \lambda^{\beta} \bar{\Pi}^{\gamma}\left[H_{\gamma \alpha \beta}-2 \alpha^{\prime} A_{\beta I} D_{\gamma} A_{\alpha I}+2 \alpha^{\prime} H_{\gamma \delta \alpha} W_{I}^{\delta} A_{\beta I}-2 \alpha^{\prime}\left(H_{\gamma \alpha}{ }^{d}+T_{\gamma \alpha}{ }^{d}\right) A_{d I} A_{\beta I}\right. \\
\left.+4 \alpha^{\prime} A_{\beta I} \widetilde{T}_{\gamma \alpha}{ }^{\delta} A_{\delta I}\right](z) .
\end{gathered}
$$

Corrections to the coupling to $d_{\gamma}$

$$
\frac{\alpha^{\prime}}{w-z} \lambda^{\alpha} \lambda^{\beta} d_{\gamma}\left[T_{\beta \alpha}{ }^{\gamma}+2 \alpha^{\prime} \widetilde{T}_{\delta \alpha}^{\gamma} W_{I}^{\delta} A_{\beta I}-2 \alpha^{\prime} \widetilde{T}_{c \alpha}{ }^{\gamma} A_{c I} A_{\beta I}-2 \alpha^{\prime} U_{I \alpha}{ }^{\gamma} A_{\beta I}\right] .
$$

Corrections to the coupling to $\bar{J}_{0}^{I}$

$$
\begin{gathered}
-\alpha^{\prime} \frac{\bar{w}-\bar{z}}{(w-z)^{2}} \lambda^{\alpha} \lambda^{\beta} \bar{J}^{I}\left[F_{\alpha \beta I}+2 \alpha^{\prime}\left(D_{(\gamma} A_{\alpha) I}+\widetilde{T}_{\gamma \alpha}^{C} A_{C I}\right) W_{J}^{\gamma} A_{\beta J}\right. \\
\left.+2 \alpha^{\prime}\left(\partial_{[c} A_{\alpha] I}+\widetilde{T}_{c \alpha}{ }^{D} A_{D I}\right) A_{c J} A_{\beta J}\right](z) .
\end{gathered}
$$

\subsection{Addition of Counter-terms}

Let's now concentrate in finding the Yang-Mills Chern-Simons 3-form by adding appropiate counter-terms. Keeping in mind the lowest order in $\alpha^{\prime}$ holomorphicity constraints $T_{\alpha b c}+T_{\alpha c b}=0=H_{\alpha b c}$; the conditions for nilpotency at one loop look like

From the coupling to $\Pi^{c}$

$$
\begin{gathered}
\lambda^{\alpha} \lambda^{\beta}\left[\left(T_{\beta \alpha}{ }^{c}+H_{\beta \alpha}^{c}\right)-4 \alpha^{\prime} A_{\beta I}\left(D_{\alpha} A_{c I}+\widetilde{T}_{\alpha c}{ }^{D} A_{D I}\right)+2 \alpha^{\prime} A_{\beta I} \partial_{c} A_{\alpha I}\right. \\
\left.-2 \alpha^{\prime} f^{I J K} A_{c I} A_{\alpha J} A_{\beta K}+2 \alpha^{\prime}\left(T_{\alpha \delta}{ }^{c}+H^{c}{ }_{\alpha \delta}\right) W_{I}^{\delta} A_{\beta I}\right](z)=0 .
\end{gathered}
$$


From the coupling to $\bar{\Pi}^{c}$

$\lambda^{\alpha} \lambda^{\beta}\left[\left(T_{\beta \alpha}{ }^{c}-H^{c}{ }_{\alpha \beta}\right)-2 \alpha^{\prime} A_{\beta I} \partial_{c} A_{\alpha I}+2 \alpha^{\prime}\left(T_{\delta \alpha}{ }^{c}-H^{c}{ }_{\delta \alpha}\right) W_{I}^{\delta} A_{\beta I}-4 \alpha^{\prime} A_{\beta I} \widetilde{T}_{c \alpha}{ }^{\gamma} A_{\gamma I}\right](z)=0$

Adding (4.66) and (4.67) gives the condition

$$
\begin{gathered}
\lambda^{\alpha} \lambda^{\beta}\left[T_{\beta \alpha}{ }^{c}-2 \alpha^{\prime} A_{\beta I}\left(D_{\alpha} A_{c I}+\widetilde{T}_{\alpha c}{ }^{D} A_{D I}\right)-\alpha^{\prime} f^{I J K} A_{c I} A_{\alpha J} A_{\beta K}+2 \alpha^{\prime} T_{\alpha \delta}{ }^{c} W_{I}^{\delta} A_{\beta I}\right. \\
\left.-2 \alpha^{\prime} A_{\beta I} \widetilde{T}_{c \alpha}{ }^{\gamma} A_{\gamma I}\right]=0 .
\end{gathered}
$$

Substracting (4.66) and (4.67) gives the condition

$$
\begin{gathered}
\lambda^{\alpha} \lambda^{\beta}\left[H_{\beta \alpha}^{c}-2 \alpha^{\prime} A_{\beta I}\left(D_{[\alpha} A_{c] I}+\widetilde{T}_{\alpha c}{ }^{D} A_{D I}\right)-\alpha^{\prime} f^{I J K} A_{c I} A_{\alpha J} A_{\beta K}+2 \alpha^{\prime} H_{\alpha \delta}{ }^{c} W_{I}^{\delta} A_{\beta I}\right. \\
\left.+2 \alpha^{\prime} A_{\beta I} \widetilde{T}_{c \alpha}^{\gamma} A_{\gamma I}\right]=0
\end{gathered}
$$

Now, suppose that a counter-term of the form $\frac{K_{1}}{2 \pi} \int d^{2} z \partial Z^{M} \bar{\partial} Z^{N} A_{N I} A_{M I}$ is added to the action, where $K_{1}$ is a constant to be determined. This amounts to redefine the space-time metric $G_{M N} \rightarrow G_{M N}+2 \alpha^{\prime} K_{1} A_{M I} A_{N I}$. The expansion of this counter-term will contain the terms

$$
\begin{gathered}
S_{C}=\frac{K_{1}}{2 \pi} \int d^{2} x\left[\partial Y^{A} \bar{\partial} Y^{B} A_{B I} A_{A I}+\partial Y^{A} \bar{\Pi}^{B} A_{B I} Y^{C}\left(\partial_{C} A_{A I}+\frac{1}{2} \widetilde{T}_{C A}^{D} A_{D I}\right)+\right. \\
\partial Y^{A} \bar{\Pi}^{B} Y^{C}\left(\partial_{C} A_{B I}+\widetilde{T}_{C B}^{D} A_{D I}\right) A_{A I}+\Pi^{A} \bar{\partial} Y^{B} A_{B I} Y^{C}\left(\partial_{C} A_{A I}+\widetilde{T}_{C A}{ }^{D} A_{D I}\right)+ \\
\left.\Pi^{A} \bar{\partial} Y^{B} Y^{C}\left(\partial_{C} A_{B I}+\frac{1}{2} \widetilde{T}_{C B}{ }^{D} A_{D I}\right) A_{A I}\right]
\end{gathered}
$$

which can be used to compute tree level diagrams contracting with $\lambda^{\alpha} \widehat{d}_{\alpha}(w) \lambda^{\beta} \widehat{d}_{\beta}(z)$. However this diagrams will contribute to the order $\alpha^{\prime 2}$, entering at the same foot as the one-loop diagrams. The result of these tree level diagram is

$$
\begin{gathered}
-\alpha^{\prime 2} K_{1} \frac{\bar{w}-\bar{z}}{(w-z)^{2}} \lambda^{\alpha} \lambda^{\beta} \bar{\Pi}^{C}\left[A_{C I}\left(D_{(\alpha} A_{\beta) I}+\widetilde{T}_{\alpha \beta}{ }^{D} A_{D I}\right)-2 A_{\beta I}\left(D_{\alpha} A_{C I}+\widetilde{T}_{\alpha D}{ }^{D} A_{D I}\right)\right](z) \\
\alpha^{\prime 2} K_{1} \frac{\lambda^{\alpha} \lambda^{\beta}}{w-z} \Pi^{C}\left[A_{C I}\left(D_{(\alpha} A_{\beta) I}+\widetilde{T}_{\alpha \beta}{ }^{D} A_{D I}\right)-2 A_{\beta I}\left(D_{\alpha} A_{C I}+\widetilde{T}_{\alpha C}{ }^{D} A_{D I}\right)\right](z) \\
+2 \alpha^{\prime 2} K_{1} \frac{\bar{w}-\bar{z}}{(w-z)^{2}} \bar{\partial} \lambda^{\alpha} \lambda^{\beta} A_{\alpha I} A_{\beta I}(z)+2 \alpha^{\prime 2} \frac{K_{1}}{w-z} \partial \lambda^{\alpha} \lambda^{\beta} A_{\alpha I} A_{\beta I}(z)
\end{gathered}
$$

Then, (4.66) and (4.67) will be modified respectively to

$$
\lambda^{\alpha} \lambda^{\beta}\left[\left(T_{\beta \alpha}{ }^{c}+H^{c}{ }_{\beta \alpha}\right)-4 \alpha^{\prime} A_{\beta I}\left(D_{\alpha} A_{c I}+\widetilde{T}_{\alpha c}{ }^{D} A_{D I}\right)+2 \alpha^{\prime} A_{\beta I} \partial_{c} A_{\alpha I}\right.
$$




$$
\begin{gathered}
-2 \alpha^{\prime} f^{I J K} A_{c I} A_{\alpha J} A_{\beta K}+2 \alpha^{\prime}\left(T_{\alpha \delta}^{c}+H_{\alpha \delta}^{c}\right) W_{I}^{\delta} A_{\beta I}+2 \alpha^{\prime} K_{1} A_{c I}\left(D_{(\alpha} A_{\beta) I}+\widetilde{T}_{\alpha \beta}{ }^{D} A_{D I}\right) \\
\left.-4 \alpha^{\prime} K_{1} A_{\beta I}\left(D_{\alpha} A_{c I}+\widetilde{T}_{\alpha c}{ }^{D} A_{D I}\right)\right](z)=0 \\
\lambda^{\alpha} \lambda^{\beta}\left[\left(T_{\beta \alpha}{ }^{c}-H^{c}{ }_{\alpha \beta}\right)-2 \alpha^{\prime} A_{\beta I} \partial_{c} A_{\alpha I}+2 \alpha^{\prime}\left(T_{\delta \alpha}{ }^{c}-H^{c}{ }_{\delta \alpha}\right) W_{I}^{\delta} A_{\beta I}\right. \\
\left.+2 \alpha^{\prime} K_{1} A_{c I}\left(D_{(\alpha} A_{\beta) I}+\widetilde{T}_{\alpha \beta}{ }^{D} A_{D I}\right)-4 \alpha^{\prime} K_{1} A_{\beta I}\left(D_{\alpha} A_{c I}+\widetilde{T}_{\alpha c}{ }^{D} A_{D I}\right)-4 \alpha^{\prime} A_{\beta I} \widetilde{T}_{c \alpha}{ }^{\gamma} A_{\gamma I}\right](z)=0
\end{gathered}
$$

One can add (4.72) with $(4.73)$ to obtain

$$
\begin{gathered}
\lambda^{\alpha} \lambda^{\beta}\left[T_{\beta \alpha}{ }^{c}-2 \alpha^{\prime} A_{\beta I}\left(D_{\alpha} A_{c I}+\widetilde{T}_{\alpha c}{ }^{D} A_{D I}\right)-\alpha^{\prime} f^{I J K} A_{c I} A_{\alpha J} A_{\beta K}+2 \alpha^{\prime} T_{\alpha \delta}{ }^{c} W_{I}^{\delta} A_{\beta I}\right. \\
\left.+2 \alpha^{\prime} K_{1} A_{c I}\left(D_{(\alpha} A_{\beta) I}+\widetilde{T}_{\alpha \beta}{ }^{D} A_{D I}\right)-4 \alpha^{\prime} K_{1} A_{\beta I}\left(D_{\alpha} A_{c I}+\widetilde{T}_{\alpha c}{ }^{D} A_{D I}\right)-2 \alpha^{\prime} A_{\beta I} \widetilde{T}_{c \alpha}{ }^{\gamma} A_{\gamma I}\right]=0 .
\end{gathered}
$$

If $K_{1}=-1 / 2$ and using the constraint $\lambda^{\alpha} \lambda^{\beta} F_{\alpha \beta I}=0$ one arrives at

$$
\lambda^{\alpha} \lambda^{\beta}\left[T_{\beta \alpha}{ }^{c}+2 \alpha^{\prime} T_{\alpha \delta}{ }^{c} W_{I}^{\delta} A_{\beta I}-2 \alpha^{\prime} A_{\beta I} \widetilde{T}_{c \alpha}^{\gamma} A_{\gamma I}\right]=0
$$

Furthermore, forming a three-level diagram with $\widehat{d}_{\alpha} Y^{\beta} \bar{\Pi}^{C} \widetilde{T}_{C \beta}{ }^{\alpha}$ and $\partial Y^{\alpha} \bar{\partial} Y^{\beta} A_{\beta I} A_{\alpha I}$ in (4.70), with precisely this value for $K_{1}$ one can cancel the term proportional to $A_{\beta I} \widetilde{T}_{c \alpha}{ }^{\gamma} A_{\gamma I}$ in (4.75) and (4.69) . Also, with this value for $K_{1}$, the counter-terms in the last line of (4.71) will cancel the contributions proportional to $\partial \lambda^{\alpha}$ and $\bar{\partial} \lambda^{\alpha}$ in (4.12) .

Note that it can be added a second counter-term of the form $\frac{K_{2}}{2 \pi} \int d^{2} z d_{\alpha} \bar{\partial} Z^{M} A_{M I} W_{I}^{\alpha}$. This amounts to redefining the supervielben $E_{M}^{\alpha} \rightarrow E_{M}^{\alpha}+\alpha^{\prime} K_{2} A_{M I} W_{I}^{\alpha}$. After expanding this counter-term, one can form a tree-level diagrams contracting it with $\frac{1}{4} \bar{\partial} Y^{\gamma} Y^{\delta} \Pi^{c}\left(T_{\delta \gamma}{ }^{c}+H_{\delta \gamma}{ }^{c}\right)$ :

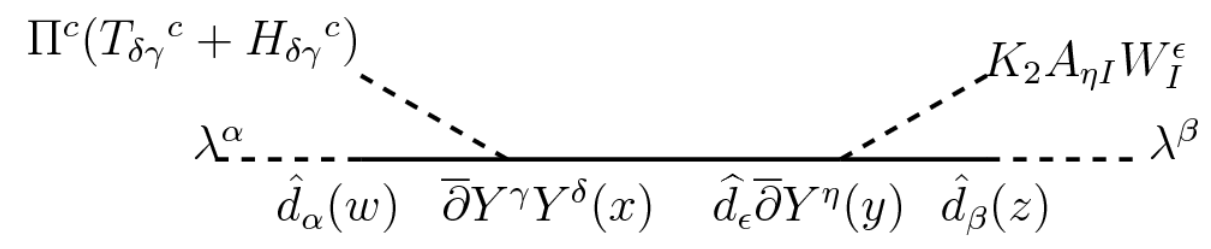

giving a contribution to the nilpotency

$$
\alpha^{\prime 2} K_{2} \frac{\lambda^{\alpha} \lambda^{\beta}}{w-z} \Pi^{c}\left(T_{\alpha \gamma}{ }^{c}+H_{\alpha \gamma}{ }^{c}\right) W_{I}^{\gamma} A_{\beta I}(z)
$$


while contractions with $\frac{1}{4} \partial Y^{\gamma} Y^{\delta} \bar{\Pi}^{c}\left(T_{\delta \gamma}{ }^{c}-H_{\delta \gamma}{ }^{c}\right)$ will form the diagram

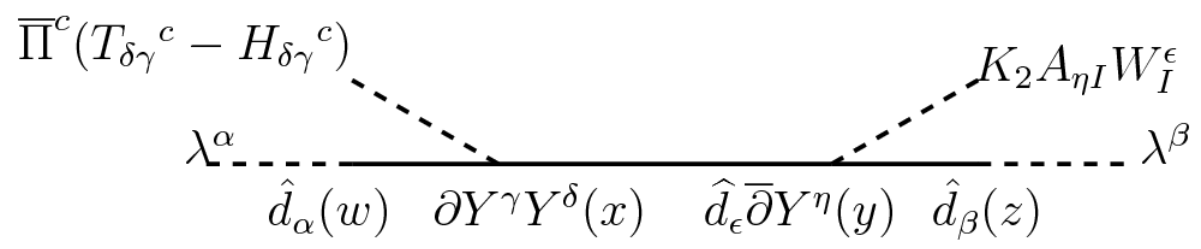

which gives the contribution

$$
-\alpha^{\prime 2} K_{2} \frac{\bar{w}-\bar{z}}{(w-z)^{2}} \lambda^{\alpha} \lambda^{\beta} \bar{\Pi}^{c}\left(T_{\alpha \gamma}^{c}-H_{\alpha \gamma}^{c}\right) W_{I}^{\gamma} A_{\beta I}
$$

It can be easily checked that for $K_{2}=-1$, adding (4.77) and (4.79) to (4.66) and (4.67) respectively; then $\lambda^{\alpha} \lambda^{\beta} T_{\alpha \beta}{ }^{c}$ will not receive $\alpha^{\prime}$ corrections, i.e. this second counter-term cancels the $\alpha^{\prime}$ correction in (4.75); while the corrections for $H_{\alpha \beta}{ }^{c}$ are

$$
\lambda^{\alpha} \lambda^{\beta}\left[H_{\beta \alpha}^{c}-2 \alpha^{\prime} A_{\beta I}\left(D_{[\alpha} A_{c] I}+\widetilde{T}_{\alpha c}^{D} A_{D I}\right)-\alpha^{\prime} f^{I J K} A_{c I} A_{\alpha J} A_{\beta K}\right]=0 .
$$

Now, the couplings to $\Pi^{\gamma}$ also receive corrections from the two counter-terms just introduced. Some of these corrections come from the coupling to $\Pi^{C}$ in (4.71) when $C$ is $\gamma$. Another correction comes from the tree-level diagram

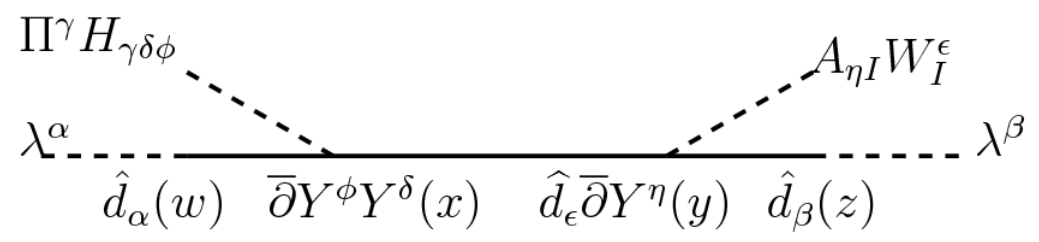

Adding those corrections and using the holomorphicity constraint $F_{\alpha \beta I}=-\frac{1}{2} W_{I}^{\gamma} H_{\gamma \alpha \beta}$, it can be checked that the $\alpha^{\prime}$ corrections to the coupling to $\Pi^{\gamma}$ are

$$
\lambda^{\alpha} \lambda^{\beta}\left[H_{\gamma \beta \alpha}-2 \alpha^{\prime} A_{\beta I}\left(D_{(\alpha} A_{\gamma) I}+\widetilde{T}_{\alpha \gamma}{ }^{D} A_{D I}\right)-\alpha^{\prime} f^{I J K} A_{\gamma I} A_{\alpha J} A_{\beta K}\right]=0
$$

Let's now identify the Chern-Simons form. It can be used the lowest order constraints in $\alpha^{\prime}$ coming from nilpotency condition $\lambda^{\alpha} \lambda^{\beta} F_{\alpha \beta I}=0$ to write (4.80) in the desired form. Since $\lambda^{\alpha} \lambda^{\beta}=\lambda^{\beta} \lambda^{\alpha}$

$$
\lambda^{\alpha} \lambda^{\beta}\left[H_{\alpha \beta}^{c}-\alpha^{\prime} \operatorname{Tr} A_{[\alpha}\left(D_{\beta} A_{c]}+\frac{1}{2} \widetilde{T}_{\beta c]}^{D} A_{D]}\right)-2 \alpha^{\prime} f^{I J K} A_{c I} A_{\alpha J} A_{\beta K}\right](z)=0
$$


Since $2 f^{I J K} A_{c I} A_{\alpha J} A_{\beta K}=\frac{2}{3} \operatorname{Tr} A_{[c} A_{\alpha} A_{\beta]}$ then

$$
\lambda^{\alpha} \lambda^{\beta}\left[H_{\alpha \beta}^{c}-\alpha^{\prime} \operatorname{Tr}\left(A_{[\alpha} D_{\beta} A_{c]}+\frac{2}{3} A_{[c} A_{\alpha} A_{\beta]}+\frac{1}{2} A_{[\alpha} \widetilde{T}_{\beta c]}^{D} A_{D}\right)\right](z)=0,
$$

which is the desired form. Similarly, (4.82) can be written as

$$
\lambda^{\alpha} \lambda^{\beta}\left[H_{\alpha \beta \gamma}-\alpha^{\prime} \operatorname{Tr}\left(A_{(\alpha} D_{\beta} A_{\gamma)}+\frac{2}{3} A_{(\gamma} A_{\alpha} A_{\beta)}+\frac{1}{2} A_{(\alpha} \widetilde{T}_{\beta \gamma)}{ }^{D} A_{D}\right)\right](z)=0 .
$$

Adding a further third counter-term $-\frac{1}{2 \pi} \int d^{2} z \lambda^{\alpha} \omega_{\beta} \bar{\partial} Z^{M} A_{M I} U_{I \alpha}{ }^{\beta}$, which amounts to redefine $\Omega_{M \alpha}{ }^{\beta} \rightarrow \Omega_{M \alpha}{ }^{\beta}-\alpha^{\prime} A_{M I} U_{I \alpha}{ }^{\beta}$; and thanks also to the other two counter-terms added, can verify that neither $\lambda^{\alpha} \lambda^{\beta} T_{\alpha \beta}{ }^{\gamma}=0$ nor $\lambda^{\alpha} \lambda^{\beta} F_{\alpha \beta I}=0$ will receive $\alpha^{\prime}$ corrections.

\section{Conclusions}

The process of finding the Yang-Mills Chern-Simons correction to the 3-superform $H$ from a string computation has been successful, in agreement with the studies of super YangMills and supergravity couplings [1], [9] and [10]. It is interesting to note that to preserve worldsheet symmetries, some redefinitions of the superfields are in order. Particularly, it was found that for the pure spinor sigma model, both $E_{M}{ }^{a}$ and $E_{M}{ }^{\alpha}$ should be redefined. The redefinition of the second one could not be found using the other descriptions for the superstring.

The procedure used in this paper is suitable for computing the Lorentz Chern-Simons 3-superform in a pretty similar way, because there is a direct analogy of the terms $\partial Z^{M} \bar{J}^{I} A_{M I}$ and $\lambda^{\alpha} \omega_{\beta} \bar{\partial} Z^{M} \Omega_{M \alpha}{ }^{\beta}$ in the action. In that case, diagrams formed by contractions of terms with three quantum fields would contribute. Work in this direction is very interesting, because a solution recently [15] has been claimed for the old debate about the inclusion of the Lorentz Chern-Simons-form in $N=1 D=10$ supergravity and the $\alpha^{\prime}$ corrections to the supergravity constraints. See [16], [17], [18 for the perturbative approach and [19], [20], [21], [22] for the non-perturbative approach. The pure spinor formalism was also used at the cohomological level in 23] to study the BRST anomaly. It would be very interesting to perform a one-loop computation to find the Lorentz Chern-Simons form, and relate the pure spinor supergravity constraints with those in [15] .

\section{Acknowledgements:}

I would like to thank Osvaldo Chandia and Vladimir Pershin for discussions and especially N. Berkovits for valuable discussions and suggestions. The work of O.B is supported by CAPES, grant 33015015001P7. 


\section{Appendix}

\subsection{Background Field Expansions}

From the expansion of the term $\frac{1}{2 \pi \alpha^{\prime}} \int d^{2} z \frac{1}{2} \partial Z^{M} \bar{\partial} Z^{N} B_{N M}$

$\frac{1}{2 \pi \alpha^{\prime}} \int d^{2} z\left[\frac{1}{2} \Pi^{B} \bar{\Pi}^{A} Y^{C} H_{C A B}+\frac{1}{4} Y^{A} \partial Y^{B} \bar{\Pi}^{C} H_{C B A}-\frac{1}{4} Y^{A} \bar{\partial} Y^{B} \Pi^{C} H_{C B A}+\frac{1}{4} Y^{A} Y^{B} \Pi^{C} \bar{\Pi}^{D} H_{D C B A}\right]$,

where $H_{A B C}=(-)^{a(b+n)+(c+p)(a+b)} 3 E_{C}^{P} E_{B}^{N} E_{A}^{M} \partial_{[M} B_{N P]}$,

$$
\partial_{[M} B_{N P]}=\frac{1}{3}\left(\partial_{M} B_{N P}+(-)^{m(n+p)} \partial_{N} B_{P M}+(-)^{p(m+n)} \partial_{p} B_{M N}\right)
$$

and $H_{D C B A}=(-)^{B(C+D)} \nabla_{B} H_{D C A}-(-)^{B C} T_{D B}{ }^{E} H_{E C A}+(-)^{D(B+C)} T_{C B}{ }^{E} H_{E D A}$.

From the expantion of $\frac{1}{2 \pi \alpha^{\prime}} \int d^{2} z \partial Z^{M} \bar{J}^{I} A_{M I}$

$$
\begin{gathered}
\frac{1}{2 \pi \alpha^{\prime}} \int d^{2} z\left[( \overline { J } _ { 0 } ^ { I } + \overline { J } _ { 1 } ^ { I } + \overline { J } _ { 2 } ^ { I } ) \left(\partial Y^{A} A_{A I}+\Pi^{A} Y^{B}\left(\partial_{B} A_{A I}+\widetilde{T}_{B A}{ }^{C} A_{C I}\right)+\Pi^{A} A_{A I}\right.\right. \\
+\frac{1}{2} \partial Y^{A} Y^{B}\left(\partial_{[B} A_{A] I}+\widetilde{T}_{B A}{ }^{C} A_{C I}\right)+\frac{1}{2} Y^{A} Y^{B} \Pi^{C} \widetilde{T}_{C B}{ }^{D}\left(\partial_{D} A_{A I}+\widetilde{T}_{D A}{ }^{E} A_{E I}\right) \\
-\frac{(-)^{B C}}{2} Y^{A} Y^{B} \Pi^{C} \partial_{B}\left(\partial_{C} A_{A I}+\widetilde{T}_{C A}{ }^{D} A_{D I}\right)
\end{gathered}
$$

From the expansion of $\frac{1}{2 \pi \alpha^{\prime}} \int d^{2} z d_{\alpha} \bar{\partial} Z^{M} E_{M}^{\alpha}$

$$
\frac{1}{2 \pi \alpha^{\prime}} \int d^{2} z\left[\left(d_{\alpha 0}+\widehat{d}_{\alpha}\right)\left(\bar{\partial} Y^{\alpha}+\bar{\Pi}^{B} Y^{C} \widetilde{T}_{C B}{ }^{\alpha}\right)\right],
$$

where the terms quadratic in $Y$ were written in (3.4).

From the expansion of $\frac{1}{2 \pi \alpha^{\prime}} \int d^{2} z d_{\alpha} \bar{J}^{I} W_{I}^{\alpha}$

$$
\frac{1}{2 \pi \alpha^{\prime}} \int d^{2} z\left[\left(d_{\alpha 0}+\widehat{d}_{\alpha}\right)\left(\bar{J}_{0}^{I}+\bar{J}_{1}^{I}+\bar{J}_{2}^{I}\right)\left(\frac{1}{2} Y^{B} Y^{C} \partial_{C} \partial_{B} W_{I}^{\alpha}+Y^{C} \partial_{C} W_{I}^{\alpha}+W_{I}^{\alpha}\right) .\right.
$$

From the expansion of $\frac{1}{2 \pi \alpha^{\prime}} \int d^{2} z \lambda^{\alpha} \omega_{\beta} \bar{\Pi}^{C} \Omega_{C \alpha}{ }^{\beta}$

$$
\begin{gathered}
\frac{1}{2 \pi \alpha^{\prime}} \int d^{2} z\left[( \widehat { \lambda } ^ { \alpha } \omega _ { \beta } + \lambda ^ { \alpha } \widehat { \omega } _ { \beta } + \widehat { \lambda } ^ { \alpha } \widehat { \omega } _ { \beta } ) \left(\frac{1}{2} \bar{\partial} Y^{D} Y^{C}\left(\partial_{[C} \Omega_{D] \alpha}{ }^{\beta}+\widetilde{T}_{C D}{ }^{E} \Omega_{E \alpha}{ }^{\beta}\right)+\bar{\Pi}^{C} \Omega_{C \alpha}{ }^{\beta}\right.\right. \\
+\frac{1}{2} Y^{C} Y^{D} \bar{\Pi}^{E} \widetilde{T}_{E D}{ }^{F}\left(\partial_{F} \Omega_{C \alpha}{ }^{\beta}+\widetilde{T}_{F C}{ }^{G} \Omega_{G \alpha}{ }^{\beta}\right)+\bar{\partial} Y^{C} \Omega_{C \alpha}{ }^{\beta}+\bar{\Pi}^{C} Y^{D}\left(\partial_{D} \Omega_{C \alpha}{ }^{\beta}+\widetilde{T}_{D C}{ }^{E} \Omega_{E \alpha}{ }^{\beta}\right) \\
\left.\left.-\frac{1}{2}(-)^{D E} Y^{C} Y^{D} \bar{\Pi}^{E} \partial_{D}\left(\partial_{E} \Omega_{C \alpha}{ }^{\beta}+\widetilde{T}_{E C}{ }^{F} \Omega_{F \alpha^{\beta}}{ }^{\beta}\right)\right)\right] .
\end{gathered}
$$

From the expansion of $\frac{1}{2 \pi \alpha^{\prime}} \int d^{2} z \lambda^{\alpha} \omega_{\beta} \bar{J}^{I} U_{I \alpha}{ }^{\beta}$

$\frac{1}{2 \pi \alpha^{\prime}} \int d^{2} z\left[\left(\lambda^{\alpha} \omega_{\beta}+\widehat{\lambda}^{\alpha} \omega_{\beta}+\lambda^{\alpha} \widehat{\omega}_{\beta}+\widehat{\lambda}^{\alpha} \widehat{\omega}_{\beta}\right)\left(\bar{J}_{0}^{I}+\bar{J}_{1}^{I}+\bar{J}_{2}^{I}\right)\left(\frac{1}{2} Y^{C} Y^{D} \partial_{D} \partial_{C} U_{I \alpha}{ }^{\beta}+Y^{C} \partial_{C} U_{I \alpha}{ }^{\beta}+U_{I \alpha}{ }^{\beta}\right)\right]$. 


\section{References}

[1] M. Green and J. Schwarz, "Anomaly Cancellations in Supersymmetric D = 10 Gauge Theory and Superstring Theory," Phys. Lett. B 149, 117 (1984)

[2] C.M Hull and E. Witten, Supersymmetric Sigma Models and the Heterotic String, Phys. Let. B160 (1985) 398.

[3] N. Berkovits, "Super-Poincare Covariant Quantization of the Superstring," JHEP 0004, 018 (2000) [arXiv: hep-th/0001035].

[4] N. Berkovits and P.S. Howe, "Ten-Dimensional Supergravity Constraints from the Pure Spinor Formalism for the Superstring", Nucl. Phys. B 635, 75 (2002) arXiv:hepth/0112160

[5] P.S. Howe, "Pure spinor lines in superspace and ten-dimensional supersymmetric theories," Phys. Lett. B258: 141 (1991).

[6] O. Chandia and B.C. Vallilo, "Conformal Invariance of the Pure Spinor Superstring in a Curved Background," JHEP 0404 (2004) 041 arXiv:hep-th/0401226].

[7] O.A. Bedoya and O. Chandia, "One-loop Conformal Invariance of the Type II Pure Spinor Superstring in a Curved Background," JHEP 0701 (2007) 042, arXiv:hepth/0609161].

[8] J. Kluson, "Note about Classical Dynamics of Pure Spinor String on $A d S_{5} \times S^{5}$ Background," Eur. Phys. J. C50:1019 (2007) arXiv:hep-th/0603228. J. Kluson "Note About Redefinition of BRST Operator for Pure Spinor String in General Background," arXiv:0803.4390 [hep-th].

[9] J. J. Atick, A. Dhar and B. Ratra, "Superspace formulation of ten-dimensional $N=1$ supergravity coupled to $N=1$ super Yang-Mills," Phys. Rev. D 33, 2824 (1986).

[10] P.S. Howe, "Pure Sinors, function superspaces and supergravity theories in ten and eleven dimensions," Phys. Lett. B273: 90 (1991).

[11] A. Sen, "Local Gauge And Lorentz Invariance Of The Heterotic String Theory," Phys. Lett. B 166: 300, (1986).

[12] C.M. Hull and P.K. Townsend, "World-sheet Supersymmetry and Anomaly Cancellation in the Heterotic String," Phys. Lett. 178: 187, (1986).

[13] O. Chandia, "A note on the Classical BRST Symmetry of the Pure Spinor String in a Curved Background," JHEP 0607 (2006) 019 arXiv:hep-th/0604115

[14] J. de Boer and K. Skenderis, "Covariant Computation of the Low Energy Effective Action of the Heterotic Superstring", Nucl. Phys. B 481, 129 (1996) [arXiv: hepth/9608078].

[15] K. Lechner and M. Tonin, "Superspace formulations of ten-dimensional supergravity," arXiv: 0802.3869[hep-th]

[16] S. Bellucci and S. J. Gates, Jr., " $\mathrm{D}=10, \mathrm{~N}=1$ Superspace Supergravity And The Lorentz Chern Simons Form," Phys. Lett.B208: 456, (1988). 
[17] S. Bellucci, D.A. Depireux and S.J. Gates Jr., "Consistent And Universal Inclusion Of The Lorentz Chern-Simons Form In D=10, N=1 Supergravity Theories," Phys. Lett. B238:315, (1990)

[18] S.J. Gates, Jr., A. Kiss and W. Merrell, "Dynamical equations from a first-order perturbative superspace formulation of $10 \mathrm{D}, \mathrm{N}=1$ string-corrected supergravity (I)," JHEP 0412:047, (2004), arXiv:hep-th/0409104

[19] R. D'Auria, P. Frè, M. Raciti and F. Riva, "Anomaly Free Supergravity In D=10.1. The Bianchi Identities And The Bosonic Lagrangian," Int. J. Mod. Phys. A3:953, (1988)

[20] M. Raciti, F. Riva and D. Zanon, "Perturbativa Approach to D=10 Superspace Supergravity With a Lorentz Chern-Simons Form," Phys. Lett. B227:118, (1989).

[21] L. Bonora, P. Pasti and M. Tonin, "Superspace Formulation Of 10-D Sugra+Sym Theory A La Green-Schwarz, " Phys. Lett. B188: 335, (1987); L. Bonora, M. Bregola, K. Lechner, P. Pasti and M. Tonin, "Anomaly Free Supergravity And Superyang-Mills Theories In Ten-Dimensions, " Nucl.Phys.B296:877, (1988).

[22] L. Bonora et al, "Some remarks on the supersymmetrization of the Lorentz ChernSimons in D=10 N=1 supergravity theories," Phys. Lett. B277:306, (1992).

[23] O. Chandia and M. Tonin, "BRST anomaly and superspace constraints of the pure spinor heterotic string in a curved background," JHEP 0709:016, 2007m arXiv: 0707.0654[hep-th]. 